\title{
Optimal clustering algorithm for wireless sensor networks using combined turbid ant swarm and improved myopic algorithm for maximizing life of sensors
}

Muthukumar S (D muthukumars2525@gmail.com )

St. Xavier's Catholic College of Engineering

D. Hevin Rajesh

St Xavier's Catholic College of Engineering

\section{Research Article}

Keywords: wireless sensor network, optimal clustering, cluster formation, cluster head, network lifetime, liveliness consumption

Posted Date: April 30th, 2021

DOl: https://doi.org/10.21203/rs.3.rs-449642/v1

License: (c) (1) This work is licensed under a Creative Commons Attribution 4.0 International License.

Read Full License 


\title{
Optimal clustering algorithm for wireless sensor networks using
}

\section{combined turbid ant swarm and improved myopic algorithm for maximizing life of sensors}

\author{
${ }^{1 *}$ Muthukumar S, ${ }^{2}$ Hevin Rajesh .D \\ ${ }^{1 *}$ Research scholar, St. Xavier's Catholic College of Engineering, Nagercoil. \\ ${ }^{2}$ Associate Professor, St. Xavier's Catholic College of Engineering, Nagercoil, Tamil Nadu, \\ India. \\ 1*Email- muthukumars2019phd@gmail.com
}

\begin{abstract}
Wireless sensor network (WSNs) consistsof a variety of sensor nodes to sense the environmentalparameters and communicate to the sink knot. The control factor is that controlling the power of the sensor nodes and charging or replacing the battery is an expensive and complicated process, which affects the sensor node lifetime as well as network lifetime. Clustering is one of the schemes that save energy by reducing the amount of intracluster communication cost. In this paper, an optimal clustering (OC) algorithm proposed to maximizes the network lifetime at data transmission without compromising energy expenditure. In OC algorithm, first we propose the turbid ant swarm(TAS) algorithm to form the clusters, which reduces much amount of energy consumption. Then, an improved myopic (IM) algorithm proposed to determines the cluster head $(\mathrm{CH})$ of cluster, which minimizes reclustering frequency and intra-communication charge. The proposed OC-TAS-IM algorithm is concentrate to get better the energy efficiency and extend the network life span. Moreover, the planned algorithm is practical to the low-energy adaptive clustering hierarchy (LEACH)
\end{abstract}


to perform the entire routing. The completion and imitation experiment with Network Simulator (NS2) are obtainable in order to authenticate our planned OC-TAS-IM algorithm. Imitation outcome illustrate that OC-TAS-IM algorithm is stable in terms of energy consumption and network lifetime because of optimal clustering.

Keywords-wireless sensor network, optimal clustering, cluster formation, cluster head, network lifetime, liveliness consumption

\section{Introduction}

Current technological advance in wireless communications, electronics, micro electro mechanical system (MEMSs), and micro-sensors have led to the expansion of short-distance, low-power, and multi-function small sensor nodes [1]. These sensors have the capability to correspond with each other or honestly with an external base. Each sensor node includes sensitivity, dispensation, transmission, mobilization, positioning, and power units [2]. To keep the price and size of these sensors small, they have small batteries that can store very little. This leads to significant limitations in the power available for communication, which reduces transmission range and data speeds [3]. A feeler on such a network can only converse straight with other sensors at other distances. To enable uninterrupted communication between sensors, the sensors create a multi-hop communication network [4]. Surveillance [5], Defense [6], Disaster Management [7], Military [8], Health and Environmental Oversight [9] WSN is a combination of low-power, processing, and communication capacity nodes used to perform a temporary operation without infrastructure or central control point. The data collected by the sensors in the cluster environment [10] are reported to the Data Processing Center in CHs. 
The final evaluation of the given parameters is determined by the processing center using $\mathrm{CHs}$ related information. The data dispensation middle can be a dedicated machine or any of these sensors. Because sensors transmit data over short distance in a cluster environment, the energy consumption of each sensor communicate directly with the data dispensation center is much less than that consumed by the network. Recently, several clustering methods have been suggested in different contexts [11] - [20]. Low-energy adaptive clustering hierarchy $(\mathrm{LEACH})$ protocol [11] is the opening step-by-step clusterbased steering protocol for determining the optimal clusters on WSN networks to save energy and improve network life. Distributed optimal-compression clustering (DOC) procedure used to solve the minimum weight set cover (MWSC) problem [12]. In a cluster sequence constructed using the DOC protocol, the problem is calculated by giving the optimal ratio to each node in the cassette, i.e. the cost of intra-cluster communication. A multi-layer clustering routing algorithm (MLCRA) [13] calculates the dual power of the terminal and the distance between the vehicle terminal and the central station. Two-level hierarchical clustering based hybrid-routing protocol (THCHP) is used in conjunction with the adaptive periodic threshold-sensitive energy efficient sensor network (APTEEN) protocol to match the accuracy of the query average response analysis [14]. The Distance aware intelligent clustering protocol (DAIC) [15] is used as the basic concept for networking near a base station and for selecting high energy $\mathrm{CHs}$. The four configuration algorithms used to obtain centrally randomly connected K-coverage [16] are responsible for selecting the lowest sensors to ensure the field's K-coverage while maintaining the correlation between the selected sensors. A fuzzy-logic-based clustering approach by expanding the energy predication used to prolong the lifetime of WSNs by evenly distributing the workload [17]. Lightweight Optimization Solution Plan based on identifying nodes in the cluster WSNs suitable for WSNs as WS helps save energy. The centralized cluster-based protocol [19], 
based on the fuzzy C-means (FCM) used for live fire detection programs, successfully enables real-time environmental action. Centralized cluster-based protocol using harmony search algorithm (HAS) [20] to detect live fires in the indoor building of the WSN test bed. WSN sensor nodes have limited processing power, communication bandwidth, and storage space, which are personal challenges in data management and information processing. The main features used to evaluate WSN performance are network longevity. The lifespan of the grid is calculated by the remaining by-power of the system, so the most important and important challenge of the WSN is the efficient use of energy resources.

\section{Contribution of the paper}

An optimal clustering (OC) algorithm is projected base on the turbid ant swarm (TAS) algorithm based cluster formation and an improved myopic (IM) algorithm based $\mathrm{CH}$ selection process. The major object of planned OC-TAS-IM algorithm used to maximize the network lifetime without compromising energy consumption.The proposed OC-TAS-IM algorithm has improved the network lifetime and makes it appropriate in a real-life submission like ambient hotness monitor for fire revealing.

The rest of the document is prepared as follows. Section 2 summarizes the related previous works and their limitations, the difficulty method and system model is describes in Section 3. Section 4 describes the projected OC-TAS-IM algorithm with the proper mathematical model. Simulation outcome are obtainable in section 5 while conclusionis given in section 6 .

\section{Related works}

Hoang et al. [21] have planned a centralized cluster-based protocol that allows us to harness the powerful computing capabilities of the base station. The harmony search optimization (HAS) algorithm is implemented within a reasonable time for live operation. The protocol is 
created and implemented using the HSA in the WSN. The HSA is activated at a base station with a system connected to the exit terminal. To create online network configuration clusters at the HSA base station and to improve the selection of CHs.

Lin et al. [22] have planned a fan-shaped clustering (FSC) technique, which divides a huge network into dense rings, divides each ring into different parts. Network-based clustering and data transfer used to divide the network into square grids are carried out by the nearest neighbor, $\mathrm{CH}$. All $\mathrm{CHs}$ are selected and sorted into a network. The collected data is then divided into a network of sync networks for a rectangular network, but in two stages. Clusters for cluster data collection created by nine consecutive low-level clusters deliberate to transfer cluster data are provided at the lowest level. The FSC method takes into account various criteria for performance study, such as total live nodes, total residual energy, and pocket accumulation speed.

Lee et al. [23] have proposed a hybrid hierarchical clustering approach (HHCA) based on a hybrid central network and scattered cluster. HHCA is base on a three - tier series that is considered to expand the previous two - tier clustering approach. This algorithm centrally defines grid heads and distributes cluster heads. The three-layer (TL) sequence, along with the number of nodes in contact with the base position, contributes to the energy gain. TLHHCA focuses on improving network performance based on network life expectancy and cluster numbers.

Huynh et al. [24] have planned a distribution cluster approach used to determine the optimal $\mathrm{CH}$ of each cluster based on energy expenditure and end-to-end delay (TED). Conventional terminals are graded together, where $\mathrm{CH}$ is selected along with the TED value for power consumption and final and final delays. The cluster versatile hop root algorithm is the value function used based on the delayed model. $\mathrm{CH}$ candidates are a group of touch nodes that send messages, and then they receive no messages or receive more power than 
messages. The multi-hop route algorithm is subject to ultimate delay control for sinking from CHs with low power costs.

Akila et al. [25] have proposed a fuzzy-based cluster for cooperative on a vague basis to reduce energy consumption problems. $\mathrm{CH}$ will be selected using residual energy, confidence, signal interference-plus-noise ratio, and load parameters. Intra-cluster routing is performed using a network index-based probability route diagram using a blur-based cluster protocol that calculates the cooperative terminal joining the cluster. particle swarm optimization (PSO) technology is used to determine the optimal path between data transfer CHs at each cooperative node.

Zhou et al. [26] have proposed Clustering a WSN reduces clustering protocol minimizes the high consumption of CHs. The advanced PSO algorithm is used to create a cluster structure, reduce transmission distances and improve grid power consumption. This eliminates inconsistencies between data messages and allows shared terminals to turn off radio components at each common relay terminal and turn off at any time other than sending or receiving messages from relay terminals. It improves energy efficiency by reducing overall consumption and energy consumption and balancing consumption and energy consumption throughout the life of the grid.

Wu et al. [27] have proposed the Leach cluster algorithm was improved by improving the node residual energy and long-distance node components. Data merging speeds will be introduced to enable cluster managers to connect data and send data to the base station before capturing data. The free space model and the multi-path -bath model gradually disappear to avoid the excess energy generated by the low energy tip. Minimum wide tree primary algorithm used to configure tree network routes, bypass $\mathrm{CH}$ and key stations, and reduce the communication value of the CHS node. 
Sasirekha et al. [28] have proposed a cluster-chain mobile agent routing (CCMAR) to improve network longevity, node current and latency measurements. The main factors affecting effective data collection are network design and routing protocol, which are classified into structured aggregation, configuration, and low aggregation. CCMAR reduces cluster chain energy loss during data transmission, which can lead to network failure. Moreover, the $\mathrm{CH}$ problem encountered by the traditional LEACH algorithm quickly transcends information and sends it to a base station based on node energy.

Bahbahani et al. [29] have proposed a cooperative clustering protocol using LEACH to improve WSN duration based on energy revenues. To ensure that any consumption related to the position of the $\mathrm{CH}$ is redistributed among the nodes, the role of the $\mathrm{CH}$ is shifted between the nodes using a duty cycle, taking into account their unique energy output capacities. To maintain the neutral action of energy when the $\mathrm{CH}$ is not working, the nodes receive a data transfer duty cycle, while additional energy is released into the pockets of the other nodes. Consumption the cross-operating TDMA scheme is used to improve the relay performance according to the optimal number of clusters based on power consumption, delay and speed usage.

Deepa et al. [30] have proposed optimized QoS-based clustering with multipath routing protocol (OQoS-CMRP) to diminish power consumption. The adapted particle swarm optimization (PSO) algorithm is used to choice the $\mathrm{CHs}$ in the zinc cover area and to create clusters to solve the energy hole problem. All target immersion algorithms are used to determine the optimal way of multifaceted hop communiqué from sync to sensors to select the next hop nodes. The surrounding robin path selection algorithm is used to transfer data via OQoS-CMRP to improve communication reliability, low latency, power consumption and network performance. 


\section{Problem methodology and system model}

\subsection{Problem methodology}

Tsai et al. [31] have planned a high-performance hyper-heuristic clustering algorithm (HHCA), which improves group formation, reduces WSN power consumption using a highquality metamorphic algorithm to balance the remaining balance energy in the calculation. A hybrid algorithm that uses all the meta-heuristic and search algorithms in the merger process will only take one meta-heuristic or search algorithm from the applicant pool for possible solution in the merger method. This method selects the $\mathrm{CHs}$ with the lowest total diffusion reserve to the first in a circle. With data transmission, the total transmission distance in a circle is increased, which can be divided into two types of $\mathrm{CH}$ base stations and $\mathrm{CH}$ sensors. Consumption HHCA offers superior routine in provisos of power consumption, but does not guarantee network performance. Network life depends on the life of single nodes and the most important metric for evaluating touch networks. However, the consumption energy grid contains a specific lifespan, which creates a high range of sensor grid usage. A network can only fulfill its purpose as long as it is considered active, but not after that. This is an indicator of the maximum use of the touch network. If the meter is used in the live analysis for placement, it may help to justify the estimated value of the grid life deployment.

The problem will overcome by proposed optimal clustering (OC)algorithm, which compromised both the network lifetime and energy consumption. The turbid ant swarm (TAS) algorithm used to creates the cluster with the help of position and velocity of nodes. An improved myopic (IM) algorithm used to computes CHs with the help of decision metrics are energy consumption, network lifetime, route cost, network load and distance. The proposed OC-TAS-IM algorithm is used to improves the energy efficiency and prolong the network duration. The main contribution of proposed OC-TAS-IM algorithm is summarized as follows: 
- In OC-TAS-IM algorithm, turbid ant swarm (TAS) algorithm used to creates the cluster using sensor node situation, velocity and ant behavior.

- $\quad$ An improved myopic (IM) algorithm used to computes $\mathrm{CHs}$ using constraints are energy consumption, network lifetime, route cost, network load and distance.

- For simulation purpose, proposed OC-TAS-IM algorithm is combined with the LEACH protocol to forward data from source-destination.

\subsection{Network model}

We estimate that a network of energy-controlled sensor nodes will be used approximately in the grid area. The sensor nodes are initially powered by full power components. Each sensor typically collects data related to other sensors and sends the associated data to the $\mathrm{CH}$ at the base station. The sensor node not only recognizes that it is its own data, but also collects and synchronizes data from other sensor nodes so that the nodes not only communicate with the base station but also communicate with other sensor nodes within range, including time nodes geographical area. Within each specific cluster, a node is periodically preferred that acts as a $\mathrm{CH}$ to communicate from the cluster to the cassette. The system model of proposed OC-TASIM algorithm with LEACHrouting is exposed in Fig. 1.

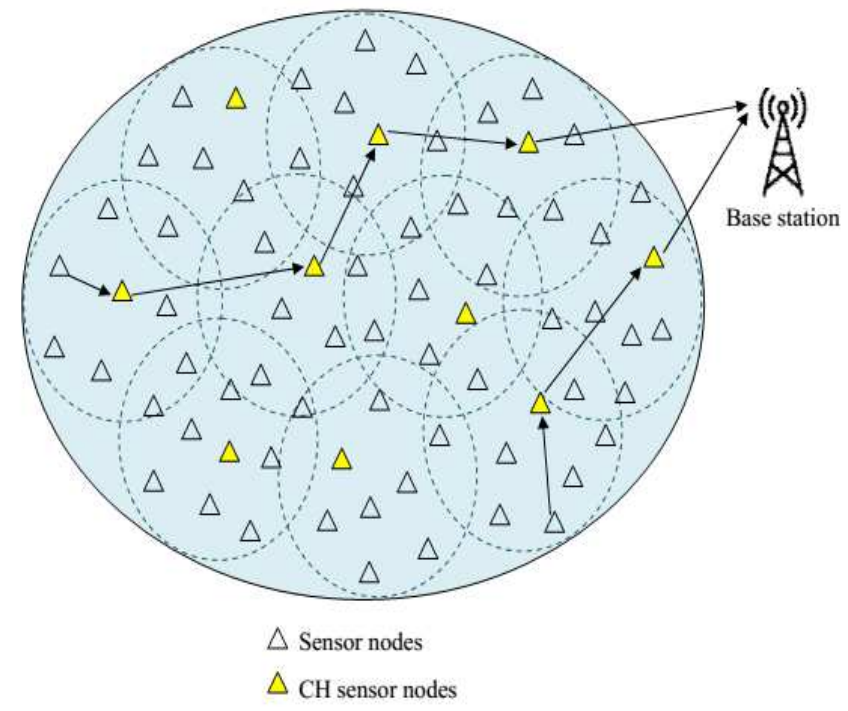

Fig. 1 System model of planned OC-TAS-IM algorithm 


\section{Proposed optimal clustering algorithm}

Generally, the clustering process consists of cluster formation and $\mathrm{CH}$ selection, which is briefly describe as follow.

\subsection{Cluster formation using turbid ant swarm (TAS) algorithm}

In WSN, clustering is the process of dividing sensor nodes into groups of similar nodes. Placing similar objects in the same cluster the same targets are in separate clusters. Cluster analysis is a difficult problem as many factors play a role in the classification of a welldeveloped clustering technique. Everyone knows that clustering systems cannot handle all types of clustering. Pre-processing the data can sometimes improve the quality of the detected clusters. One problem with clustering methods is that clusters are difficult to interpret. Also, algorithms always transfer data to clusters even when there are no clusters in the data. Therefore, if the goal is to generate assumptions about its cluster structure, it is necessary to analyze whether the data set shows a clustering trend. Ant colony optimizations (ACO) [32] solve many unique optimization problems. ACO works with artificial systems, inspired by the behavior of real ants used to solve unique optimization problems. The idea is that there is a direct link between ants through chemical pheromone testing, which helps to find shortcuts between nests and food.

Ants communicate with each other through the remnants of pheromones. The chemical that ants deposit is pheromones, which act as an important means of communication between ants, thus leading to the resolution of the next movement. When more ants find a specific path, this path (trail) becomes more attractive and other ants establish their own pheromone and follow. This auto catalyst and the combined character set the shortcut. Ants find the shortest path due to the intensity of the pheromone placed in different pathways. ACO-based clustering methods are based on the incubation sort nature of ants. As shown in 
Figure 2, the data components are scattered in an approximately two-dimensional phase. Initially, each data object in a multi-dimensional shape is distributed at approximately 2D intervals. Each ant moves randomly to this point and the data units drop. The decision to select or abandon an object is random, but it quickly affects the data of nearby ants. If the ants are surrounded by nearby data, the risk of the object falling increases. Conversely, if the data object is surrounded by different data or there is no data near it, the likelihood of selecting an object increases. In this way clustering of 2D grid elements is obtained.

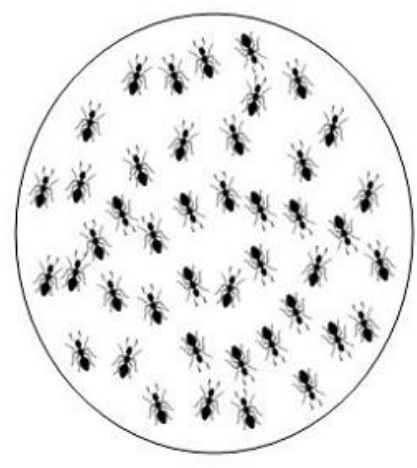

(a)

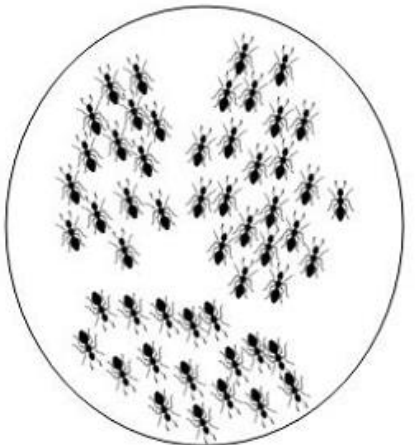

(b)

Fig. 2 ACO-based cluster formation (a) initial population (b) after cluster formation

Here, the turbid ant swarm (TAS) algorithm is inspired by the advanced ACO search criteria [32]. Additionally, the TAS algorithm is ideal for use in WSN clustering. The steps of the TAS algorithm are similar to the steps of the ACO based cluster algorithm.

When searching for food, ants can switch in sequence with each other by release pheromones. Assume that the quantity of pheromone at large by the ant is low, the pheromone remaining at the edge $[j, i]$ is usually a long path and a short path. In the beginning, we can start with ants. During evolution, a gram of ant g can conclude its next city i from the modern city $\mathrm{j}$ using the subsequent formula: 


$$
q_{j i}^{g}(s)=\left\{\begin{array}{c}
\frac{\left[\tau_{j i}(s)\right]^{\alpha}\left[\eta_{j i}(s)\right]^{\beta}}{\sum\left[\tau_{j t}(s)\right]^{\alpha}\left[\eta_{j t}(s)\right]^{\beta}}, i, t \in \text { allowed }_{g} \\
0, \quad \text { otherwise }
\end{array}\right.
$$

Where $q_{j i}^{g}(s)$ is the probability, this is a recurring number $\tau_{j i}(s)$ the amount of pheromone levels [j, i]; $\alpha$ and $\beta$ are two hyper-parameters that reflect the magnitude of trial superiority and visibility; allowed $\mathrm{k}_{\mathrm{k}}$ city are contained in the village of permitted ant $\mathrm{g}$; and $\eta_{j i}(s)$ is heuristic thing as equation (2)

$$
\eta_{j i}(s)=\frac{1}{D_{j i}}
$$

where $D_{j i}$ is the detachment or the charge of edge $[\mathrm{j}, \mathrm{i}]$ and sij are achieved by the following equation

$$
\tau_{j i}(s+1)=\rho \tau_{j i}(s)+\Delta \tau_{j i}(s, s+1)
$$

where $\rho$ is the disappearance coefficient. $\Delta \tau_{j i}(s, s+1)$ can be planned by the succeeding equations

$$
\begin{gathered}
\Delta \tau_{j i}(s, s+1)=\sum_{g=1}^{g} \tau_{j i}^{g}(s, s+1) \\
\tau_{j i}^{g}(s, s+1)=\left\{\begin{array}{cc}
\frac{P}{M_{g}}, \text { if ant } g \text { passed throughedge }[j, i] \\
0, & \text { otherwise }
\end{array}\right.
\end{gathered}
$$

where $\mathrm{P}$ is total quantity of pheromone, $\mathrm{Mg}_{\mathrm{g}}$ is total span of the tour (route) $\mathrm{g}$, and $\mathrm{G}$ is the amount of ants. In general, the typical TAS-ACO replica of TSP can be summarizing as Algorithm 1. The complex TSP time required for a convinced numeral of ACO cycle has been exceeded. In this editorial we will try to diminish the operation time of the algorithm 
based on the following improvements. If the best solution to the following equation cannot be found according to the equation, the pheromones can be updated periodically a better explanation as under equation.

$$
\tau_{j i}(s+1)=\left\{\begin{array}{l}
\rho \times \tau_{j i}(s)+\Delta \tau_{j i}(s, s+1), \\
\text { if a better solutionis obtained } \\
\tau_{j i}(s), \quad \text { otherwise }
\end{array}\right.
$$

where $\tau_{j i}(s, s+1)$ can be compute as equations (4) and (5). If the algorithm get a explanation among healthier fitness after $\mathrm{N}$ iterations, the pheromone update formula can be printed as

$$
\tau_{j i}(s+N)=\rho \times \tau_{j i 1}(s)+\tau_{j i}(s) *
$$

Where $\tau_{j i 1}(s)$ is the amount of pheromone collected from equations (3) to (5). $\tau_{j i}(s) *$ is the main method of Extra boost from "Elite Ants", the best tour in the world for ants. This is called "elitist strategy". He can calculate the equation underneath

$$
\tau_{j i}^{g}(s, s+1)=\left\{\begin{array}{cc}
\frac{P}{M_{g}}, \text { if }[j, i] \text { is in the best route } \\
0, \quad \text { otherwise }
\end{array}\right.
$$

where $M_{g}$ is the duration of the internationally best tour. Commonly presented confusing maps comprise a logistic map, tent map, Chebyshev map, and more. Compare to logistics and tent maps with allocation ranges from 0 to 1 , the Sephishev map has a wider allocation from 1 to 1 . In this article, two Chebyshev chaotic uses two confusing situations to create random factors $R_{1}(S)$ and $R_{2}(S)$. The Chebyshev map model with degree $\mathrm{N}$ can be established as follow

$$
S_{N}(Y)=\cos (N * \arccos y)(-1 \leq y \leq 1)
$$

Then, we can attain the repeated equations as under 


$$
\begin{gathered}
S_{0}(Y)=1 \\
S_{1}(Y)=1 \\
S_{2}(Y)=2 y^{2}-1 \\
S_{N+1}(Y)=2 y S_{N}(y)-S_{N-1}(y) \quad(N \geq 2)
\end{gathered}
$$

A new formula for updating speed can be written as follows

$$
V_{j D}(S+1)=\omega V_{j D}(S)+b_{1}\left|B h 1_{(s)}\left(y_{0}\right)\right|\left(q b e s t_{j D}-y_{j D}(s)\right)+b_{2}\left|B h 2_{(s)}\left(y_{0}\right)\right|\left(q b e s t_{D}-y_{j D}(s)\right)
$$

\section{Algorithm 1 TAS-ACO}

Input : Hyper parameters of TAS; Information of TSP nodes

Output : $Q_{j}$ and $V_{j}$

1. While $\mathrm{m}<\mathrm{M}$ DO

2. ACO is invoked by $\mathrm{TAS}, \mathrm{j}=0$;

3. While $\mathrm{j}<\mathrm{L}$ DO

4. $\quad \mathrm{N}=0$;

5. While $\mathrm{n}<\mathrm{NDO}$

6. ACO solves TSP by asynchronous method;

7. $\quad \mathrm{N}=\mathrm{N}+1$

8. The achieved TSP result is used to evaluate $Q_{J}$

9. Update the velocity of TAS-ACO

10. $\mathrm{J}=\mathrm{j}+1$;

11. $\mathrm{M}=\mathrm{M}+1$

12. return The optimal solution of TSP 


\subsection{Cluster head (CH) selection using improved myopic (IM) algorithm}

After cluster formation, the $\mathrm{CH}$ is selected through various controls such as energy consumption, network lifetime, route cost, network load and distance between node stations.

\subsubsection{Constraints for $\mathrm{CH}$ selection}

\section{a) Energy model}

The power model ensures optimal presentation by surroundings the functionality of each node to help turn the time ON and OFF period. In general, the spending of sensor nodes depends on the power burning up of the processing unit and the command of the communication unit. The dispensation unit chains three functional states, which are sleep, passive, and operating transition levels. The permanent state liveliness is denote as,

$$
F_{r}=\sum_{l=1}^{p} Q_{r}(l) O(l)
$$

As shown in the manual for specifying the processing unit, where is the power $Q_{r}(l)$, it is the time $O_{r}(l)$ interval of the statistically variable state $\mathrm{k}$, which can be calculated in the energy model of this processing unit. State change is defined as energy consumption is defined as,

$$
F_{r o}=\sum_{l=1}^{q} Q_{e}(l) f_{d}(l)
$$

State change consumption where and how often does the $N_{e}(l)$ frequency of state change occur in $f_{b}(l)$ energy consumption. The energy spending of dispensation unit is

$$
F_{Q}=F_{r}+F_{r o}
$$

Communiqué unit consumption energy expenditure model $\mathrm{n}$ calculates the energy required to send a bit sachet $d$.

$$
F_{o u}(q, c)=q F_{o s}+q \varepsilon_{A m p} c^{m j}
$$


Where $F_{o s}$ the energy required for a small processing of the communication unit, pl but the loss path of the path and $\varepsilon_{\text {amp }}$ the scattering of the receiver energy dissipation denotes as,

$$
\varepsilon_{A m p}=\frac{S Q_{e} Q_{0}\left(\frac{4 \pi}{\lambda}\right) m j}{g_{k} O_{E f f} S_{c}}
$$

Where $S Q_{e}$ the receiver's noise is, $Q_{0}$ is the density of the sound energy spectrum, $\mathrm{BW}$ is the channel's sound conductor, $\lambda$ is the wavelength, $g_{k}$ is the antenna gain, $O_{E f f}$ is the transmitter competence, and $S_{c}$ data rate channel. As in the multipath case as follows,

$$
F_{o u}^{P}(Q, q, c)=Q F_{\text {ou }}(q, c)
$$

The $\mathrm{q}$ bits are similar to the power transmission mode required to receive and process a packet

$$
F_{s u}(q, c)=F_{o u}(q, c)
$$

Similarly, the energy consumption of the receiving process is called a multifaceted case

$$
F_{s u}^{P}(Q, q, c)=Q F_{s u}(q, c)
$$

Finally, the total power expenditure of the sensor terminal is obtained as follow:

$$
F_{O}=F_{m}+2 Q F_{o u}(q, c)
$$

\section{b) Network lifetime}

The life of a WSN depends on the figure of energetic sensors and connections in the network, so liveliness must be used professionally to prolong the life of the network. In particular, 
when a great number of feeler nodes are used in this area, a node has specialized neighbors with sensitive devices similar to a network to deal with the failure of certain nodes. Therefore, the first node is not the only meter that measures the life expectancy of a network. As a result, node partial lifetime is a very successful metric when evaluate node concert on high-density screens. Because node density affects network connectivity, life expectancy based on the proportion of defined dead nodes reflects other definition of life in terms of connectivity and reporting. The lifetime of the sensor $\mathrm{L}$, depends on the maximum number of packet that the sensor can send to the sink. This can be ignored when analyzing sensor life as the packet transmission time is very short.

$$
o_{k}=\sum_{l=1}^{\left[m_{k}\right]} \lambda^{m_{1}} \frac{u^{m_{1}-1} f^{-\lambda \tau}}{\Gamma\left(m_{k}\right)}
$$

The $m_{k}$ sensor indicates where the greatest number of packets L can transmit through time $\tau$. Then, the lifetime of the complex follow:

$$
Q J O=O\left[\operatorname{Max}\left(o_{k}\right) \in \frac{Q_{b}}{Q}\right]
$$

Where $\mathrm{Q}$ is the integer of sensors in the complex and the number of live nodes $\mathrm{Qb}$

\section{c) Routing cost}

The route cost $(\mathrm{RC})$ between two nodes are distinct as follow,

$$
S D(q, c)=\sum_{k, l \in(q, \cup, c)} \operatorname{Cost}_{k, l}
$$

where $\operatorname{Cost}_{k, l}$ cost function for a link among nodes $\mathrm{i}$ and $\mathrm{j}$. Thus,

$$
\operatorname{Cost}_{k, l}=F_{q}+2 Q F_{o u}(q, c)+f^{\frac{1}{F_{s}^{\prime}}}
$$

where $E_{R}^{i}$ is cost meaning that take into thoughtfulness the enduring force of sensors for the energy equilibrium amid sensors.

\section{d) Network load}


The network load $\left(\mathrm{L}_{\mathrm{nw}}\right)$ is prejudiced by the queue duration and the load of person nodes in this queue and predictable as follows,

$$
J_{q z}=Q_{k}+Q_{l}+\left(j_{s f} \times Q_{c_{l}}\right)
$$

where $P_{i}$ is the amount of packet in Ni's queue, $P_{j}$ amount of packet in $\mathrm{Nj}$ 's queue, $l_{r e}$ is the retransmitting boundary of a single packet and $P_{d_{i}}$ represent the package drop by Ni due to Extreme retransmissions.

\section{e) Distance between sensor nodes to base station}

Each sensor terminal evaluates the distance (d) in terms of signal strength (SS) to the base station, which determines the power output emitted by each active sensor terminal.

$$
R R=\frac{\left(F_{O}(\alpha \beta)+o_{k}\right) \mu^{2}}{4 \mu C^{2}}
$$

where $\alpha$ is the transmitter gain, $\beta$ is the receiver gain, $\mu$ is the wavelength and $D$ is the sum of coldness between sensor nodes to base station.

$$
C=\sum_{k=1}^{i} c_{k}
$$

\subsubsection{Improved myopic (IM) algorithm}

The myopic optimization models [33] are used for imperfect forethought and short-term focus of speculation decision makers, leading to further realistic outcome. The view window considers accurate information for certain years, and information beyond this period is not available. This go ahead to a continuous decision-making procedure using the telescope rolling window, i.e. telescope window investment planning, which is edited and disseminated as soon as new information is received. However, it is impossible to repay the investment made at this stage. The myopic edition of the Global Energy organism mold used to determine distant vision is delaying investment in new technology, foremost to greater speculation requirements and greater dependence on fossil fuel in the future. Myopic models 
are an important complement to perfect foresight (PF) optimization model. Where PF models are used to determine the finest change path, myopic model versions can provide more realistic predictions about the possible conditions of electrical system evolution. Additionally, the two approaches can be used in parallel to determine the performance and effectiveness of convinced strategy tools. A significant advantage is that only minor changes to the model are required to use only the myopic model version to create the best and most possible scenes in a single frame. Another salutation advantage of the myopic sculpt is that the short-term horizon reduces the computational value. The problem is represented by the myopic principle, which is the natural heuristic principle, which selects the functional set of conditions $O_{B}$ that will increase the expected reward in the next stages.

$$
\Pi^{G}(R) \in \operatorname{ArgMax}_{b \in R_{B}} F\left[S\left(R, R_{B}\right)\right]
$$

Such policies are adopted but ignore the evolution of the system and the impact of current election results on awards in future countries. The set $O_{B}$ of specific myopic problem is significantly expanded. This set represents a polynomial scale representation and effectively solves the problem of increasing myopia. The problem of aggravating myopia is difficult, but it has optimal access to the oracle. The advanced myopic algorithm is inspired by the traditional method [33], the main purpose of which is to identify random reduction problems, for which myopic heuristics ensure a consistent optimization factor. We use an improved myopic approach to the $\mathrm{CH}$ sample process with multiple controls such as power consumption, grid lifetime, routing cost, grid load and distance. The innovative myopic heuristic reward that occurs when selecting a function in some states is different from the old one. In this obvious case, the expected reward under myopic heuristics is equivalent to the predictable repayment underneath myopic heuristics for the resultant model path. The presentation of enhanced myopic heuristics can be compared to the operation of an most favorable clear algorithm to know the understanding of Pt process. Since the Optimal Clear 
Policy lasts longer than the Optimal Clear Policy, it is sufficient to accept presentation guarantees related to the best Clear rule. Thus,

$$
R_{B}=\left(\operatorname{Min}\left(F_{O}, S D, J_{q z}, C\right) \cup \operatorname{Max}\left(O_{q z}\right): 0<o<O\right)
$$

We will only consider such an optimal policy in its continuation; indicating the order of any most approving policy or charge occupation indicates the optimal policy and values for the obvious problem. Now, the sensor node with the best most favorable worth in the bunch set (calculated from equation (27)) is selected as $\mathrm{CH}$ node.

$$
D H_{k}=\sum_{k=1}^{q} R_{B_{k}}
$$

Finally, the Brown purpose [34] used to crosscheck the elected $\mathrm{CH}$ node as follow:

$$
E\left(R_{B}\right)=\sum_{k=1}^{q}\left(R_{B_{k}}^{2}\right)^{\left(R_{B_{j+1}}^{2}\right)}+\left(R_{B_{k+1}}^{2}\right)^{\left(R_{B_{j}}^{2}\right)}
$$

\section{Simulation Results}

Network Simulator (NS-2) [35], is used to simulate the proposed OC-TAS-IM algorithm. The simulation software platform is PC with $2.67 \mathrm{GHz}$ Intel Core i3 CPU 4GB of reminiscence organization Ubuntu14.04. The entire programs for the experiment are printed in $\mathrm{C}$ and compile using TCL. The simulation environment is as follows: the sensor node number of the entire networks is $100-500$ (variable) with in $1000 \times 1000 \mathrm{~m}^{2}$ network size. The initial energy of the sensor nodes is $0.5 \mathrm{~J}$. The constant bit serial (CBR) traffic source with 512 bytes of data is used for this experiment.The transmission range of sensor nodes is 250 meters. The transmission and receiving power of sensor node is $50 \mathrm{~nJ} / \mathrm{bits}$. The performance of proposed OC-TAS-IM algorithm is analyze by the two dissimilar testing scenarios: crash of node density and impact of iteration levels (rounds). The summary of simulation parameters and scenario are given in Table 1 and 2 correspondingly. 


\begin{tabular}{|l|l|}
\hline Parameters & Values \\
\hline Number of sensor nodes & $100,200,300,400,500$ \\
\hline Network size & $1000 \times 1000 \mathrm{~m}^{2}$ \\
\hline MAC protocol & IEEE 802.11 \\
\hline Transmission range & $250 \mathrm{~m}$ \\
\hline Traffic source & CBR \\
\hline Packet size & 512 bytes \\
\hline Initial energy of sensor nodes & $0.5 \mathrm{~J}$ \\
\hline Transmission power & $50 \mathrm{n} / \mathrm{bits}$ \\
\hline Receiving power & $50 \mathrm{~nJ} / \mathrm{bits}$ \\
\hline Simulation time & 100 seconds \\
\hline
\end{tabular}

Table 2: Test scenarios

\begin{tabular}{|l|l|l|}
\hline Scenarios & Number of nodes & Number of rounds \\
\hline 1 & $\begin{array}{l}100,200,300,400, \\
500\end{array}$ & 1000 \\
\hline 2 & 500 & $\begin{array}{l}500,1000,1500,2000, \\
2500\end{array}$ \\
\hline
\end{tabular}

The proposed OC-TAS-IM algorithm is combined with LEACH protocol for further routing process through $\mathrm{CHs}$. The performance of proposed OC-TAS-IM with LEACH routing protocol is compared with the existing HHCA with LEACH protocol [31] in terms of delay, energy consumption, number of alive nodes, network lifetime, overhead and throughput. Thus,

- $\quad$ Event delay is the time it takes to dip this item into the first pocket.

- Energy consumption is the amount of energy inspired by the nodes to broadcast the data packets to the receiver. 
- The number of alive nodes is the count of number of nodes alive up to entire simulation time.

- Network lifetime is the period from the start of the network operation to the moment when the first sensorin the network runs out of energy.

- Network overhead is the total number of traffic induced during simulation time.

- Network throughput is the rate of unbeaten message delivery over a communication channel.

\subsection{Impact of node density}

In this section, we will set the optimization algorithm rounds as 1000 and unreliable the number of nodes as $100,200,300,400$ and 500 with in $1000 \times 1000 \mathrm{~m}^{2}$ network size. The outcome show that the parameter make some difference but crash is small. Fig. 3 shows the delay comparison of both planned OC-TAS-IM and existing HHCA protocol. The plot clearly depicts the delay of OC-TAS-IM protocol is very low compare to HHCA protocol. Fig. 4 shows the energy consumption comparison of both planned OC-TAS-IM and existing HHCA protocol. The plot plainly depicts the energy consumption of OC-TAS-IM protocol is very low compare to HHCA protocol.

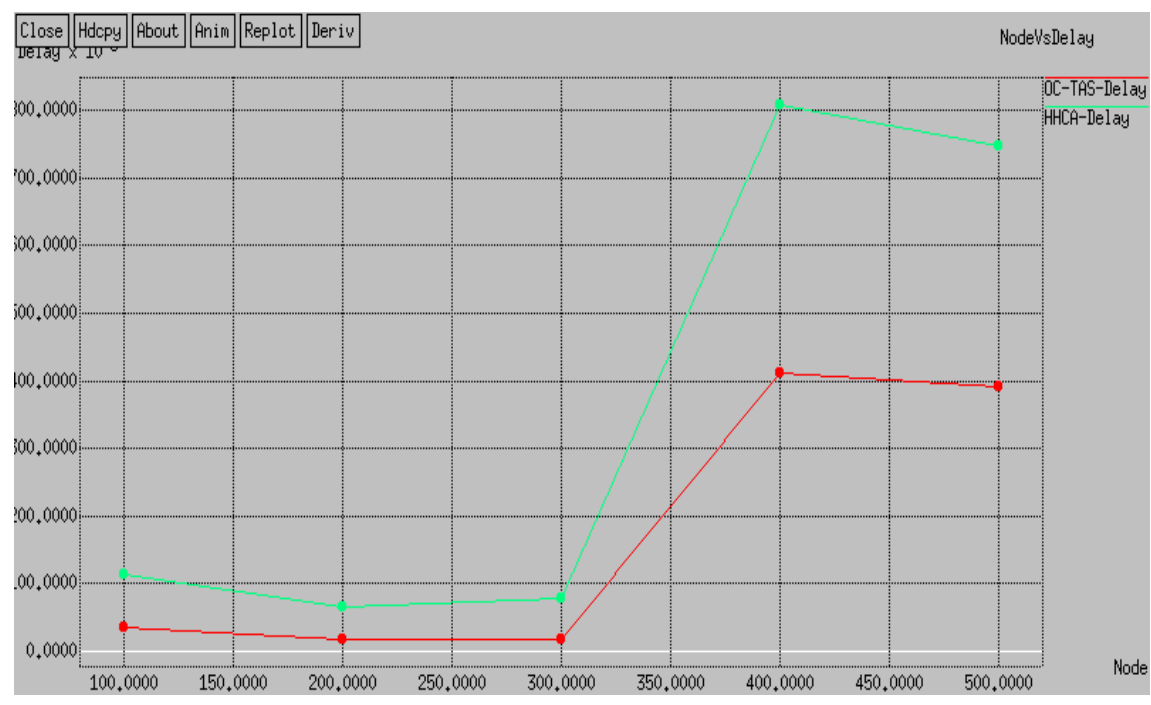

Fig. 3 Delay comparison with node density 


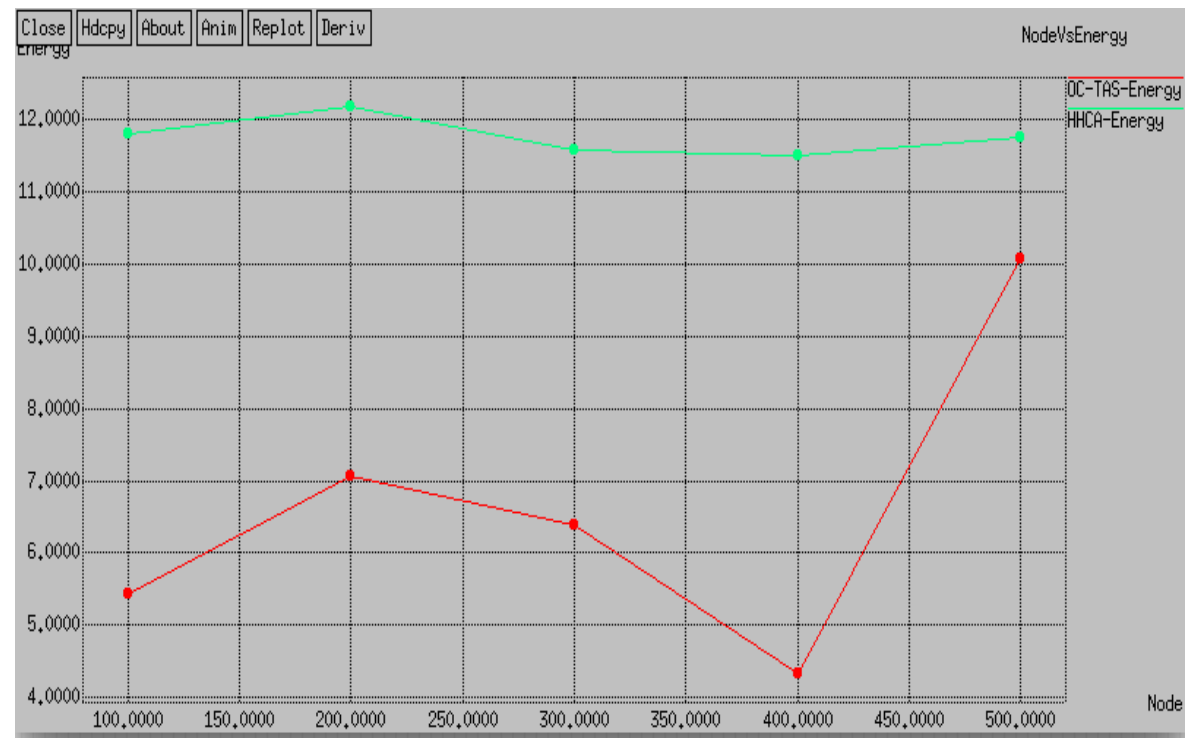

Fig. 4 Energy consumption comparison with node density

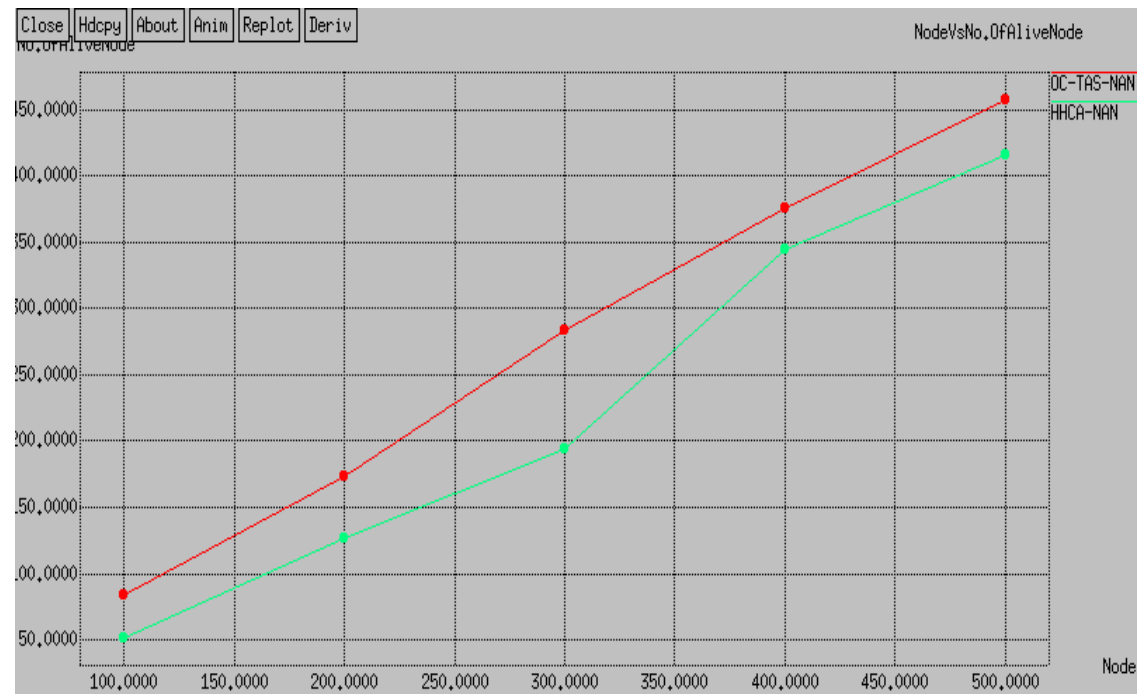

Fig. 5 Number of alive comparison with node density

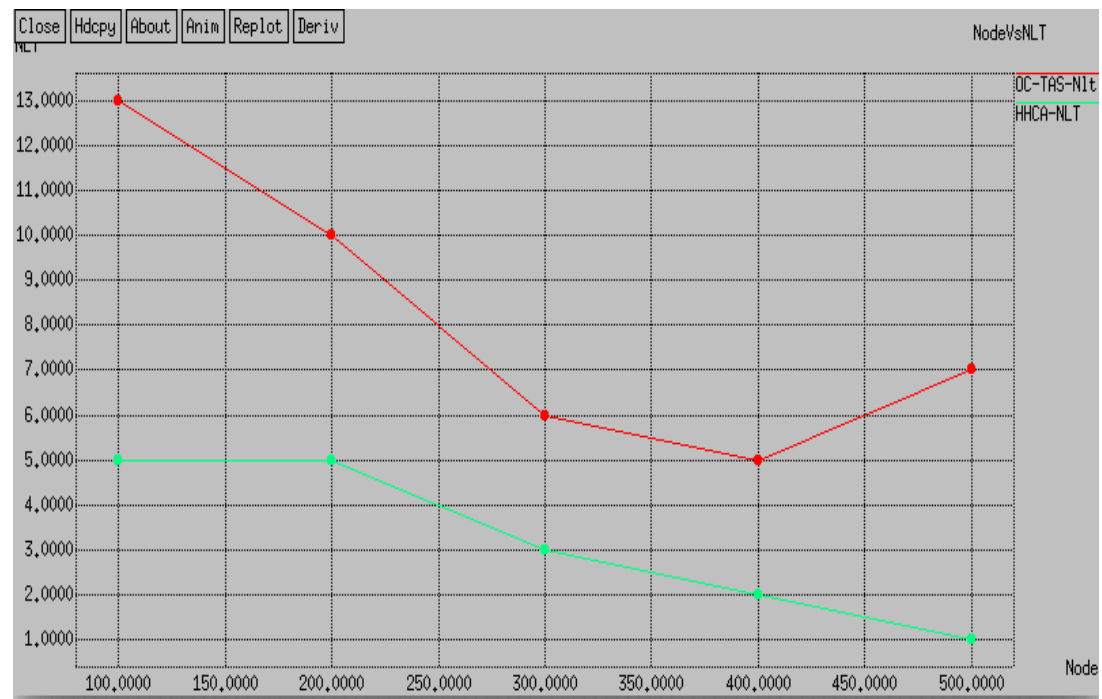


Fig. 6 Network lifetime with node density

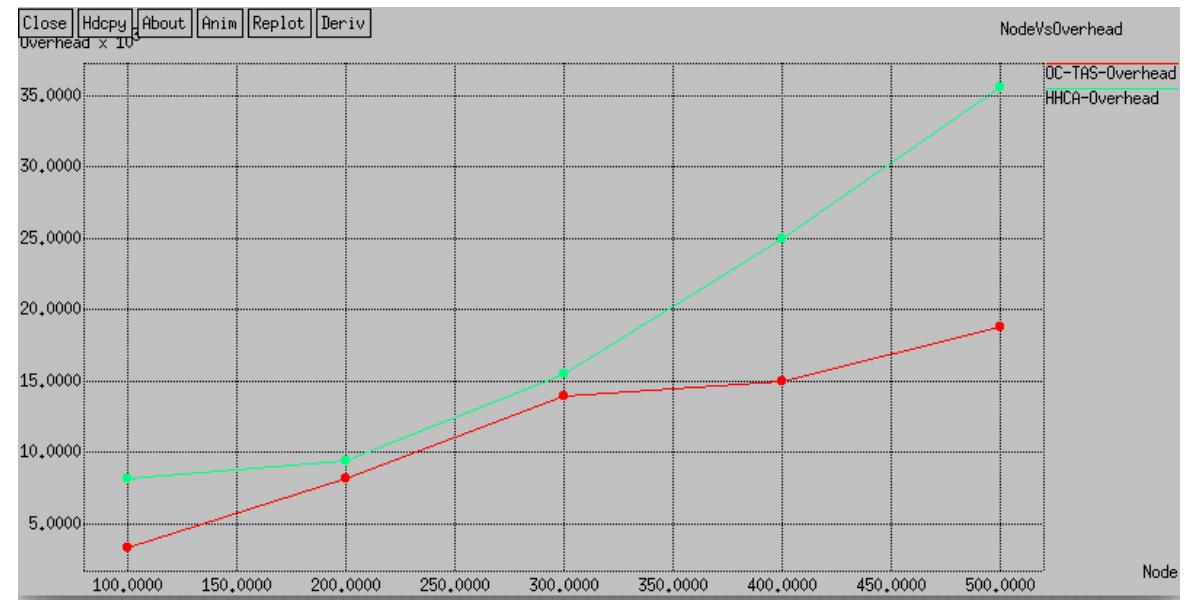

Fig. 7 Network overhead with node density

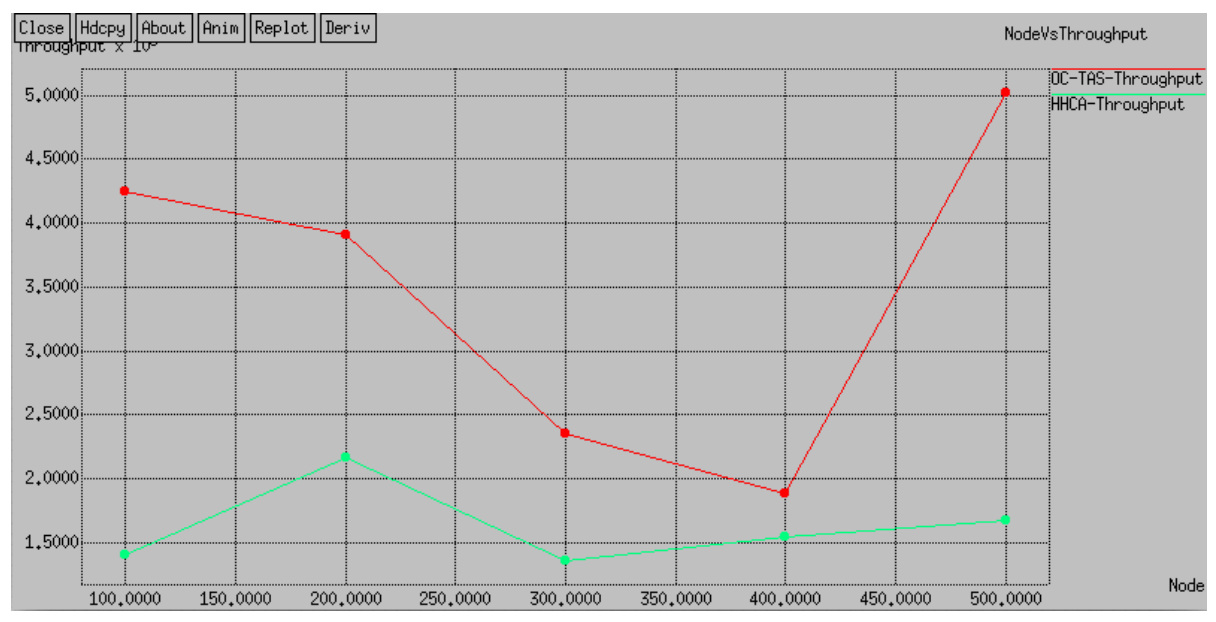

Fig. 8 Network throughput with node density

Fig. 5 shows the digit of alive nodes of both projected OC-TAS-IM and existing HHCA protocol. The plot obviously depicts the digit of alive nodes of OC-TAS-IM protocol is very high compare to HHCA protocol. Fig. 6 shows the network lifetime of both proposed OC-TAS-IM and existing HHCA protocol. The plot clearly depicts the network lifetime of OC-TAS-IM protocol is very high compare to HHCA protocol. Fig. 7 shows the network overhead of both planned OC-TAS-IM and existing HHCA protocol. The plot clearly depicts the overhead of OC-TAS-IM protocol is very low compare to HHCA protocol. Fig. 8 shows the network throughput of both planned OC-TAS-IM and obtainable HHCA protocol. The 
plot obviously depict the throughput of OC-TAS-IM protocol is very high compare to HHCA protocol.

\subsection{Impact of rounds}

In this section, we will set the sensor node as 500 and varying the iteration levels (rounds) as 500, 1000, 1500, 2000 and 2500. The results show that the parameters create some difference but collision is small. Fig. 9 shows the delay contrast of both planned OC-TAS-IM and existing HHCA protocol. The plot clearly depict the delay of OC-TAS-IM protocol is very low in terms of varying iteration levels compare to HHCA protocol. Fig. 10 shows the energy burning up comparison of both planned OC-TAS-IM and existing HHCA protocol. The plot visibly depicts the energy consumption of OC-TAS-IM protocol is very low in terms of varying iteration levels compare to HHCA protocol. Fig. 11 shows the digit of alive nodes comparison of both planned OC-TAS-IM and existing HHCA protocol. The plot clearly depicts the number of alive nodes of OC-TAS-IM protocol is very high in terms of varying iteration levels compare to HHCA protocol.Fig. 12 shows the network lifetime comparison of both planned OC-TAS-IM and existing HHCA protocol. The plot clearly depicts the network lifetime of OC-TAS-IM protocol is very high in terms of varying iteration levels compare to HHCA protocol. Fig. 13 show the network slide comparison of both planned OC-TAS-IM and existing HHCA protocol. The plot obviously depicts the network in the clouds of OCTAS-IM protocol is very low in terms of varying iteration levels compare to HHCA protocol. Fig. 14 shows the network throughput comparison of both planned OC-TAS-IM and existing HHCA protocol. The plot plainly depicts the throughput of OC-TAS-IM protocol is very high in terms of varying iteration levels compare to HHCA protocol. 


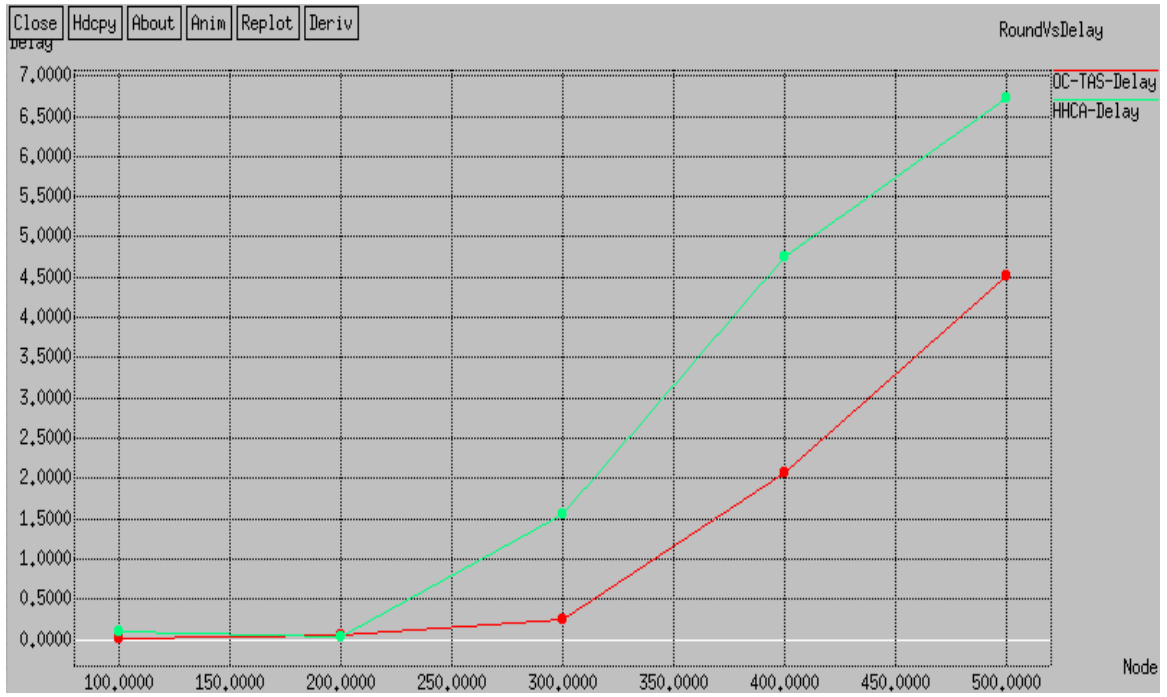

Fig. 9 Delay comparison with number of rounds

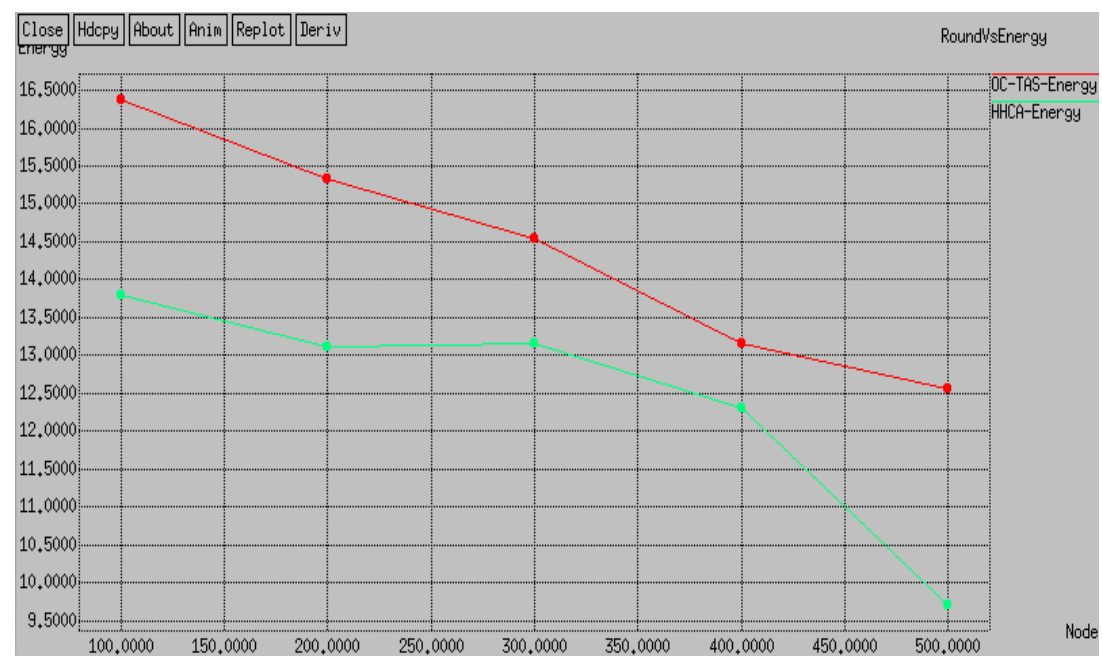

Fig. 10 Energy consumption comparison with number of rounds

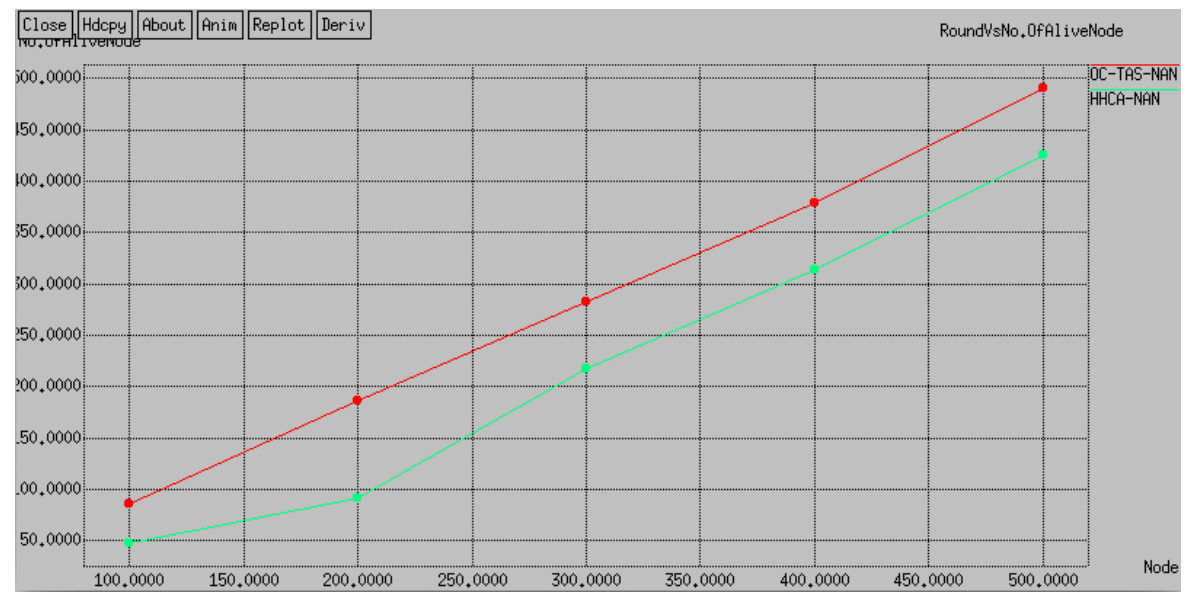

Fig. 11 Number of alive nodes comparison with number of rounds 


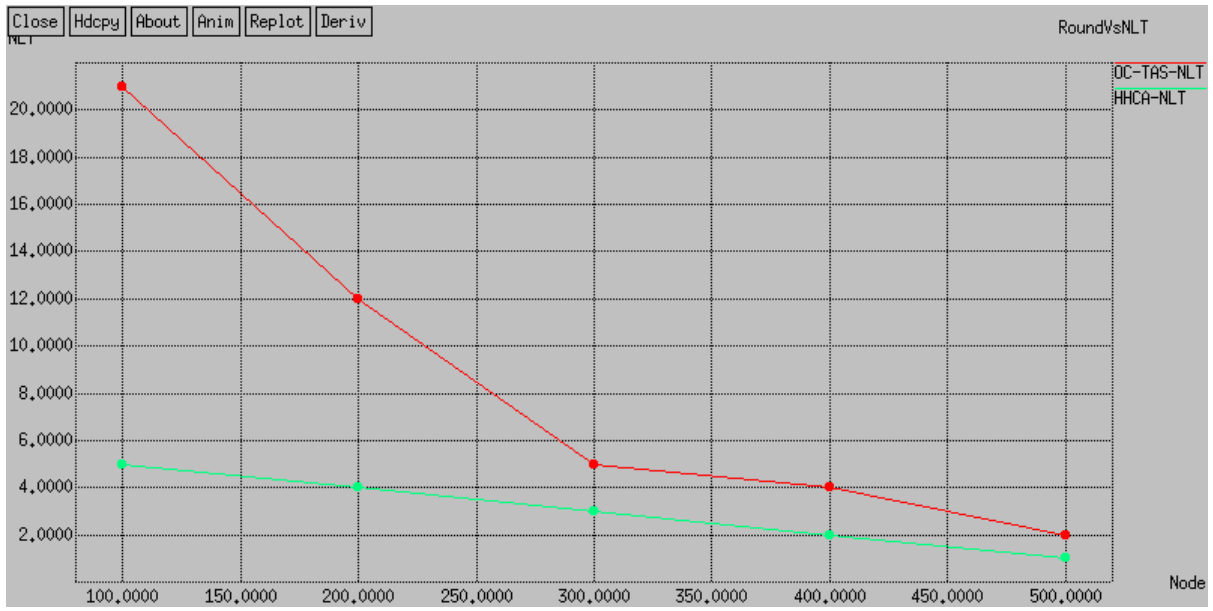

Fig. 12 Network lifetime comparison with number of rounds

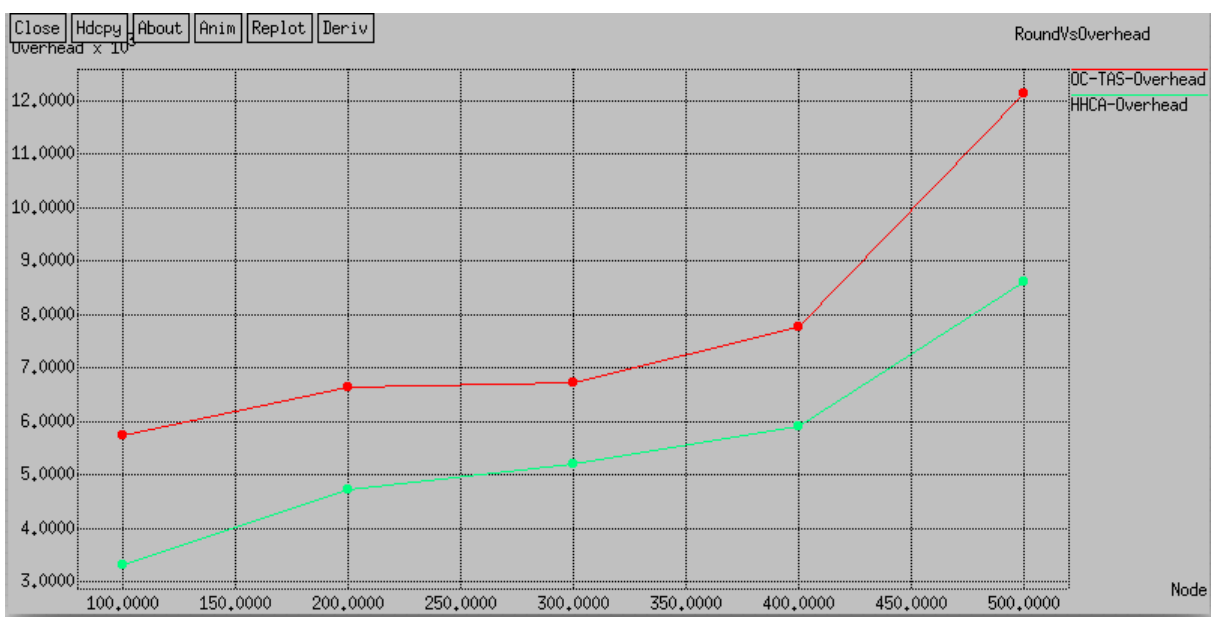

Fig. 13 Network overhead comparison with number of rounds

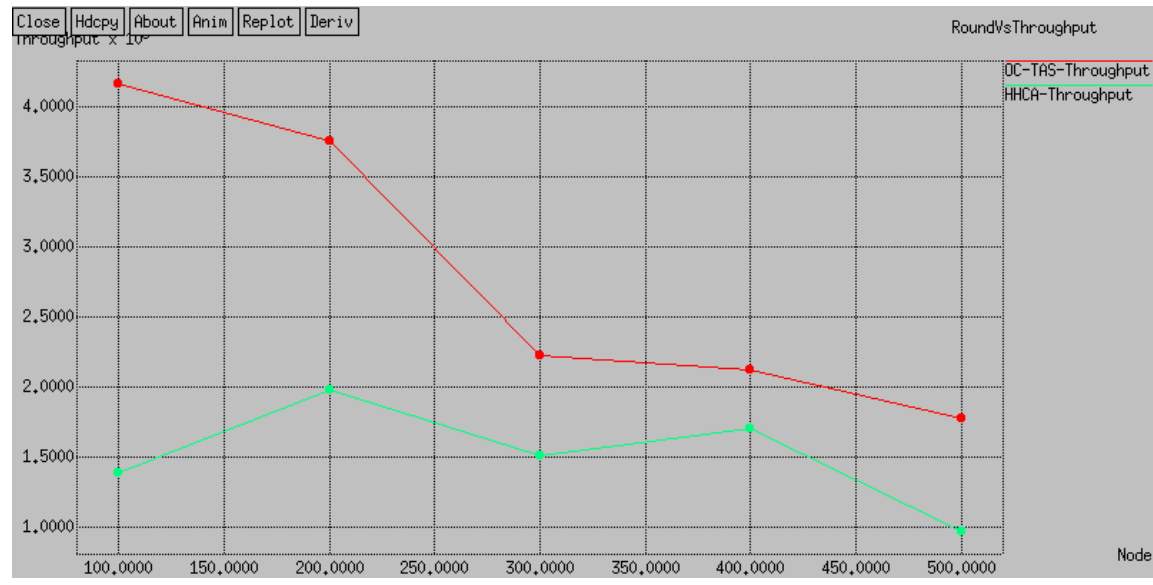

Fig. 14 Network throughput comparison with number of rounds

\section{Conclusions}


In this research, we have proposed an optimal clustering algorithm i.e. OC-TAS-IM to performs cluster and selects $\mathrm{CH}$ to maximize network lifetime without compromising energy consumption. The turbid ant swarm (TAS) algorithm used to achieves balanced cluster and improved myopic (IM) algorithm used to selects $\mathrm{CH}$ among multiple nodes in the cluster. For routing, the OC-TAS-IM algorithm follows the conventional $\mathrm{LEACH}$ through $\mathrm{CH}$ for efficient data transmission.Using simulation, we have shown the outstanding performance of OC-TAS-IM by comparing with others. We have also indicated the optimal parameter values to trade-off between energy consumption and network lifetime in a specific network size.

\section{Declaration:}

\section{Funding}

We have no funding

Conflicts of interest/Competing interests

We declare that the authors have no funding

\section{Availability of data and material}

We have no data availability

\section{References}

1. A. Sabato, C. Niezrecki and G. Fortino, "Wireless MEMS-Based Accelerometer Sensor Boards for Structural Vibration Monitoring: A Review", IEEE Sensors Journal, vol. 17, no. 2, pp. 226-235, 2017.

2. M. Kumar, R. Tripathi and S. Tiwari, "Critical data real-time routing in industrial wireless sensor networks", IET Wireless Sensor Systems, vol. 6, no. 4, pp. 144-150, 2016. 
3. N. Huynh, V. Robu, D. Flynn, S. Rowland and G. Coapes, "Design and demonstration of a wireless sensor network platform for substation asset management", CIRED - Open Access Proceedings Journal, vol. 2017, no. 1, pp. 105-108, 2017.

4. Y. Sarikaya, C. Koksal and O. Ercetin, "Dynamic Network Control for Confidential Multi-Hop Communications", IEEE/ACM Transactions on Networking, vol. 24, no. 2, pp. 1181-1195, 2016.

5. J. Zhang, G. Song, G. Qiao, T. Meng and H. Sun, "An indoor security system with a jumping robot as the surveillance terminal", IEEE Transactions on Consumer Electronics, vol. 57, no. 4, pp. 1774-1781, 2011.

6. S. Jokhio, I. Jokhio and A. Kemp, "Light-weight framework for security-sensitive wireless sensor networks applications", IET Wireless Sensor Systems, vol. 3, no. 4, pp. 298-306, 2013.

7. A. Malatras, A. Asgari and T. Bauge, "Web Enabled Wireless Sensor Networks for Facilities Management", IEEE Systems Journal, vol. 2, no. 4, pp. 500-512, 2008.

8. S. Misra, A. Singh, S. Chatterjee and M. Obaidat, "Mils-Cloud: A Sensor-Cloud-Based Architecture for the Integration of Military Tri-Services Operations and Decision Making", IEEE Systems Journal, vol. 10, no. 2, pp. 628-636, 2016.

9. N. Dey, A. Ashour, F. Shi, S. Fong and R. Sherratt, "Developing residential wireless sensor networks for ECG healthcare monitoring", IEEE Transactions on Consumer Electronics, vol. 63, no. 4, pp. 442-449, 2017.

10. C. Moraes and D. Har, "Charging Distributed Sensor Nodes Exploiting Clustering and Energy Trading", IEEE Sensors Journal, vol. 17, no. 2, pp. 546-555, 2017.

11. S. Singh, P. Kumar and J. Singh, "A Survey on Successors of LEACH Protocol", IEEE Access, vol. 5, pp. 4298-4328, 2017. 
12. Jun Zheng, Pu Wang and Cheng Li, "Distributed Data Aggregation Using Slepian-Wolf Coding in Cluster-Based Wireless Sensor Networks", IEEE Transactions on Vehicular Technology, vol. 59, no. 5, pp. 2564-2574, 2010.

13. Y. Liu, N. Xiong, Y. Zhao, A. Vasilakos, J. Gao and Y. Jia, "Multi-layer clustering routing algorithm for wireless vehicular sensor networks", IET Communications, vol. 4, no. 7 , p. $810,2010$.

14. S. Muruganathan, A. Sesay and W. Krzymien, "Analytical query response time evaluation for a two-level clustering hierarchy based wireless sensor network routing protocol", IEEE Communications Letters, vol. 14, no. 5, pp. 486-488, 2010.

15. N. Gautam and J. Pyun, "Distance aware intelligent clustering protocol for wireless sensor networks", Journal of Communications and Networks, vol. 12, no. 2, pp. 122-129, 2010.

16. H. Ammari and S. Das, "Centralized and Clustered k-Coverage Protocols for Wireless Sensor Networks", IEEE Transactions on Computers, vol. 61, no. 1, pp. 118-133, 2012.

17. J. Lee and W. Cheng, "Fuzzy-Logic-Based Clustering Approach for Wireless Sensor Networks Using Energy Predication", IEEE Sensors Journal, vol. 12, no. 9, pp. 28912897, 2012.

18. X. Li, F. Zhou and J. Du, "LDTS: A Lightweight and Dependable Trust System for Clustered Wireless Sensor Networks", IEEE Transactions on Information Forensics and Security, vol. 8, no. 6, pp. 924-935, 2013.

19. D. Hoang, R. Kumar and S. Panda, "Realisation of a cluster-based protocol using fuzzy C-means algorithm for wireless sensor networks", IET Wireless Sensor Systems, vol. 3, no. 3, pp. 163-171, 2013.

20. O. Alia, "A dynamic harmony search-based fuzzy clustering protocol for energy-efficient wireless sensor networks", Annals of Telecommunications, 2017. 
21. D. Hoang, P. Yadav, R. Kumar and S. Panda, "Real-Time Implementation of a Harmony Search Algorithm-Based Clustering Protocol for Energy-Efficient Wireless Sensor Networks", IEEE Transactions on Industrial Informatics, vol. 10, no. 1, pp. 774-783, 2014.

22. H. Lin, L. Wang and R. Kong, "Energy Efficient Clustering Protocol for Large-Scale Sensor Networks", IEEE Sensors Journal, vol. 15, no. 12, pp. 7150-7160, 2015.

23. J. Lee and T. Kao, "An Improved Three-Layer Low-Energy Adaptive Clustering Hierarchy for Wireless Sensor Networks", IEEE Internet of Things Journal, vol. 3, no. 6, pp. 951-958, 2016.

24. X. WANG, X. ZHANG and G. CHEN, "Delay-Constrained and Energy-Efficient CrossLayer Routing in Wireless Sensor Networks", Journal of Software, vol. 22, no. 7, pp. 1626-1640, 2011.

25. I. Akila and R. Venkatesan, "A Fuzzy Based Energy-aware Clustering Architecture for Cooperative Communication in WSN", The Computer Journal, vol. 59, no. 10, pp. 1551$1562,2016$.

26. Y. Zhou, N. Wang and W. Xiang, "Clustering Hierarchy Protocol in Wireless Sensor Networks Using an Improved PSO Algorithm", IEEE Access, vol. 5, pp. 2241-2253, 2017.

27. W. Wu, N. Xiong and C. Wu, "Improved clustering algorithm based on energy consumption in wireless sensor networks", IET Networks, vol. 6, no. 3, pp. 47-53, 2017.

28. S. Sasirekha and S. Swamynathan, "Cluster-chain mobile agent routing algorithm for efficient data aggregation in wireless sensor network," in Journal of Communications and Networks, vol. 19, no. 4, pp. 392-401, August 2017. 
29. M. Bahbahani and E. Alsusa, "A Cooperative Clustering Protocol With Duty Cycling for Energy Harvesting Enabled Wireless Sensor Networks", IEEE Transactions on Wireless Communications, vol. 17, no. 1, pp. 101-111, 2018.

30. O. Deepa and J. Suguna, "An optimized QoS-based clustering with multipath routing protocol for Wireless Sensor Networks", Journal of King Saud University - Computer and Information Sciences, 2017.

31. C. Tsai, W. Chang, K. Hu and M. Chiang, "An Improved Hyper-Heuristic Clustering Algorithm for Wireless Sensor Networks", Mobile Networks and Applications, vol. 22, no. 5, pp. 943-958, 2017.

32. C. Pessoa, C. Ranzan, L. Trierweiler and J. Trierweiler, "Development of Ant Colony Optimization (ACO) Algorithms Based on Statistical Analysis and Hypothesis Testing for Variable Selection", IFAC-PapersOnLine, vol. 48, no. 8, pp. 900-905, 2015.

33. M. Karimzadeh-Farshbafan and F. Ashtiani, "Semi-myopic algorithm for resource allocation in wireless body area networks", IET Wireless Sensor Systems, vol. 8, no. 1, pp. 26-35, 2018.

34. O. Begambre and J. Laier, "A hybrid Particle Swarm Optimization - Simplex algorithm (PSOS) for structural damage identification", Advances in Engineering Software, vol. 40, no. 9, pp. 883-891, 2009.

35. T. Issariyakul and E. Hossain, "Introduction to Network Simulator NS2", 2012. 
Figures

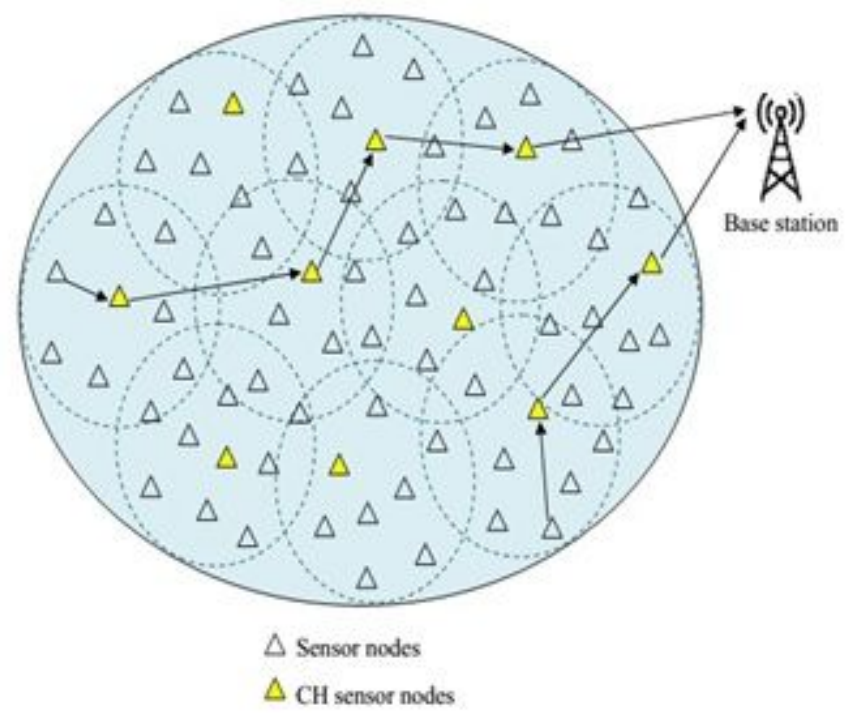

Figure 1

System model of planned OC-TAS-IM algorithm

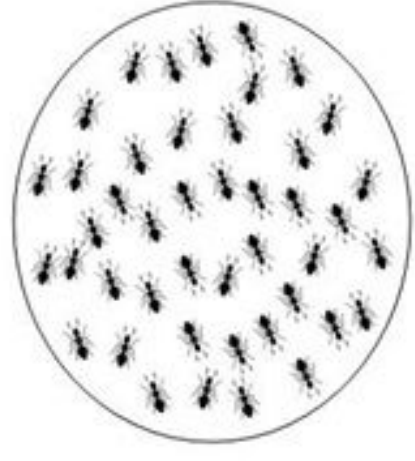

(a)

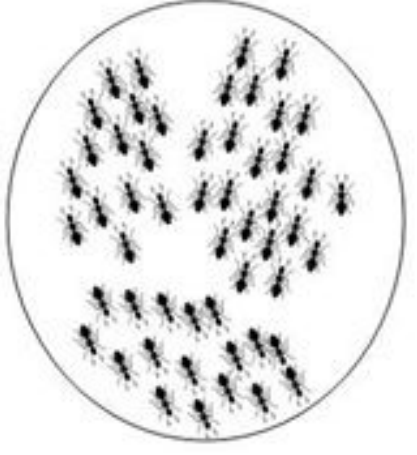

(b)

Figure 2

ACO-based cluster formation (a) initial population (b) after cluster formation 


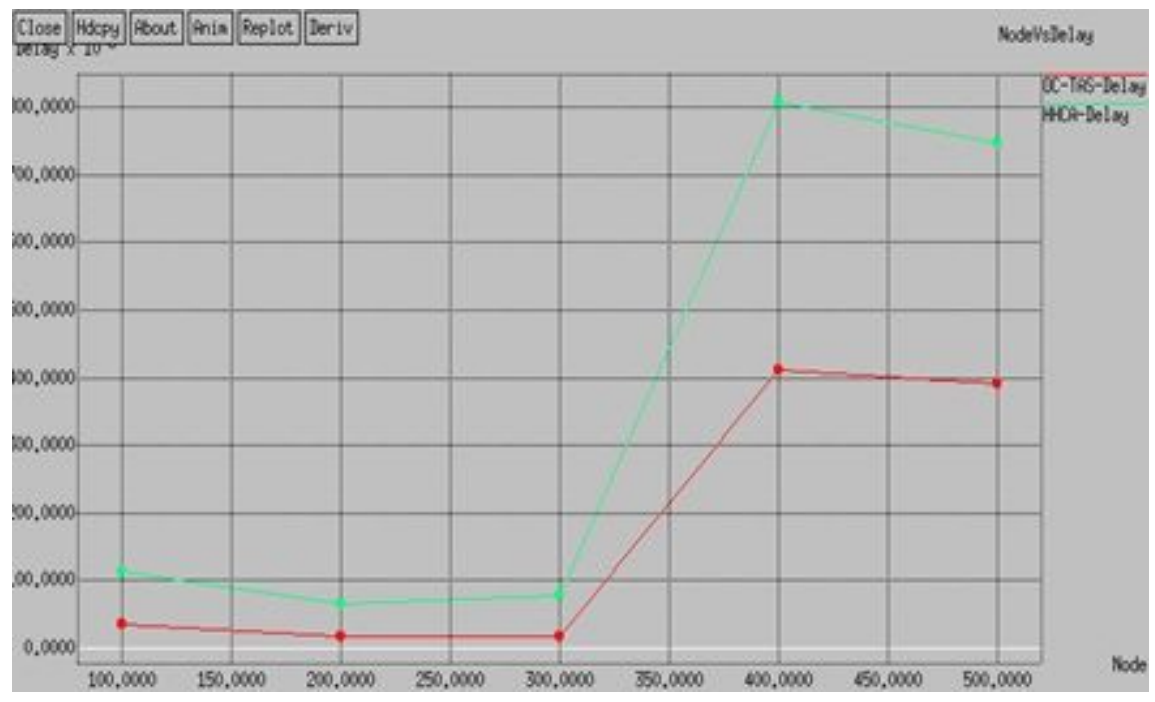

\section{Figure 3}

Delay comparison with node density

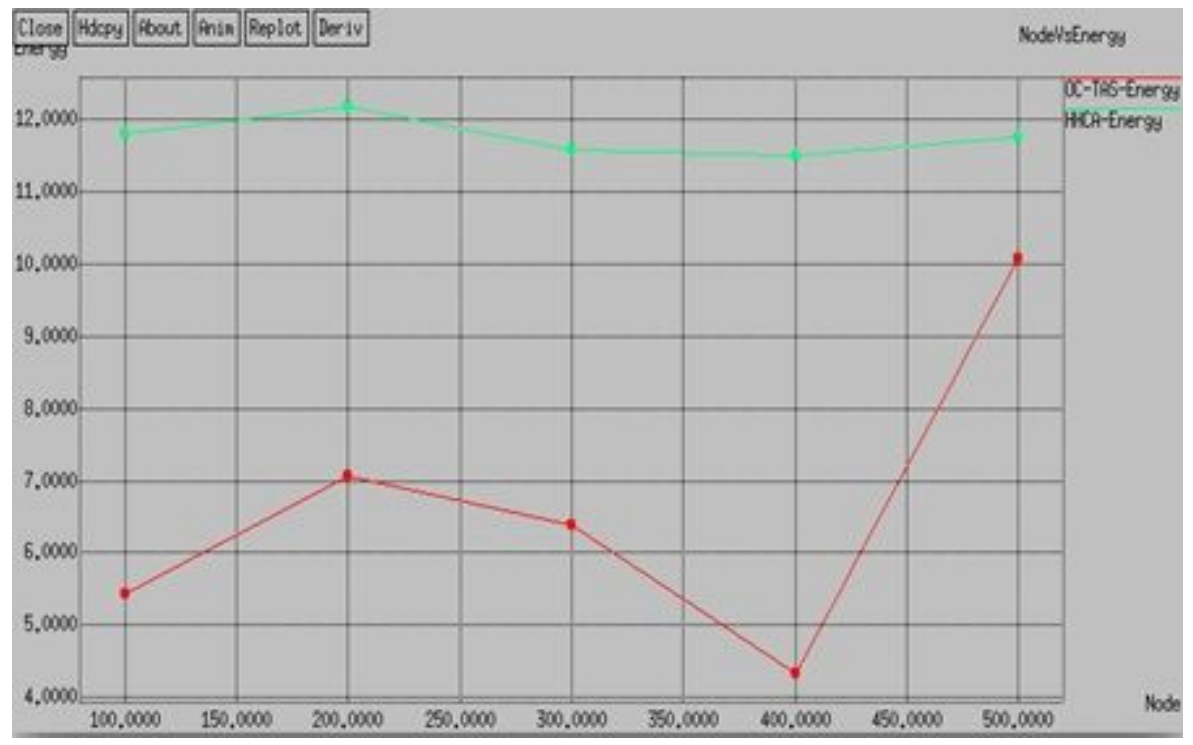

\section{Figure 4}

Energy consumption comparison with node density 


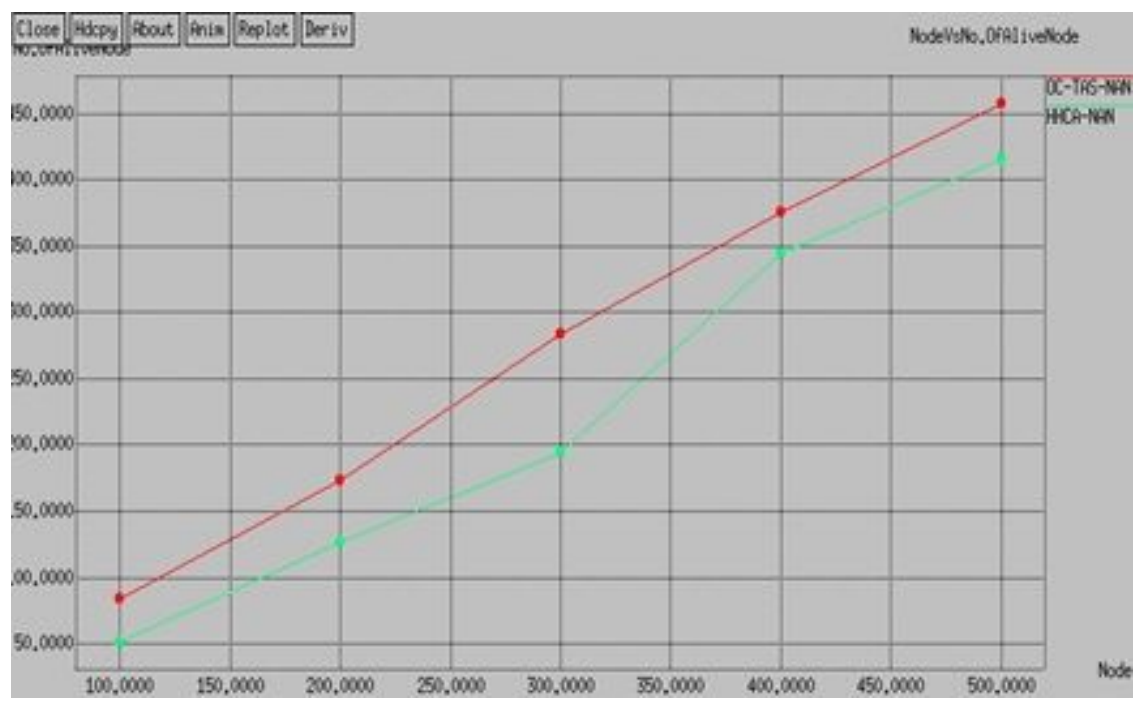

\section{Figure 5}

Number of alive comparison with node density

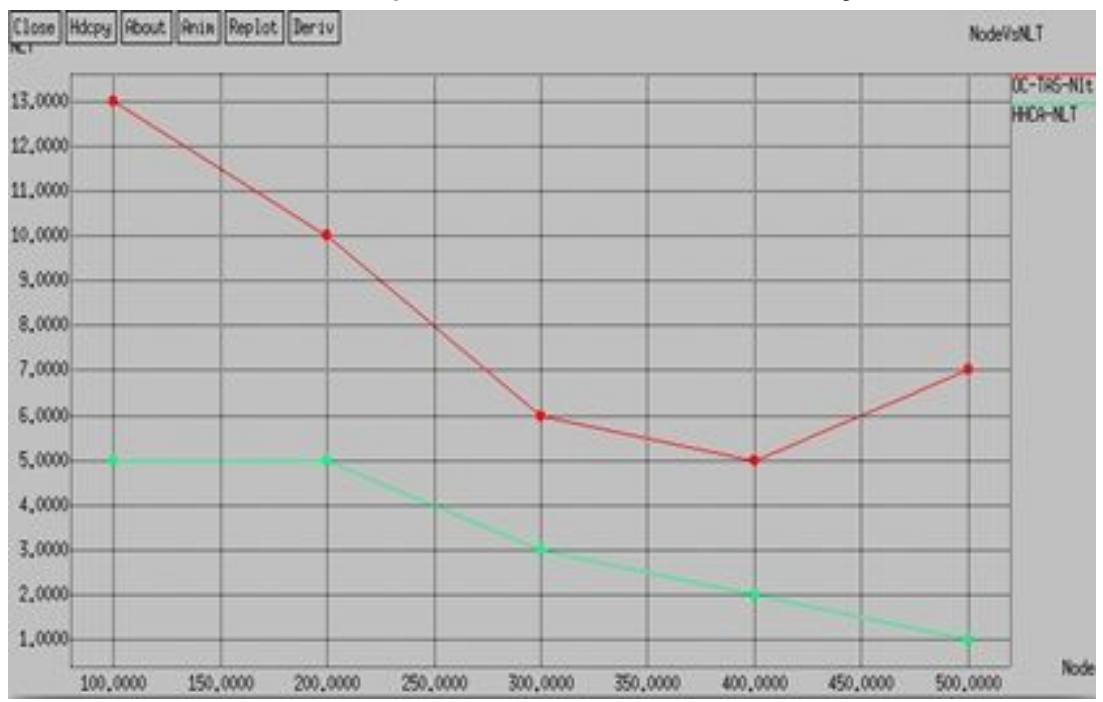

\section{Figure 6}

Network lifetime with node density

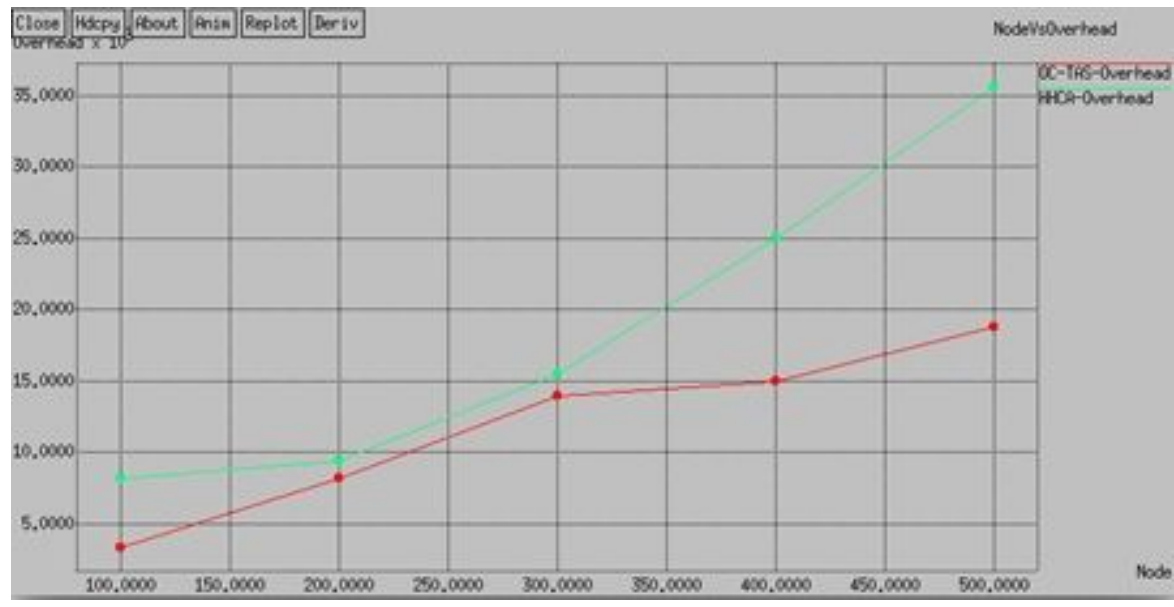


Figure 7

Network overhead with node density

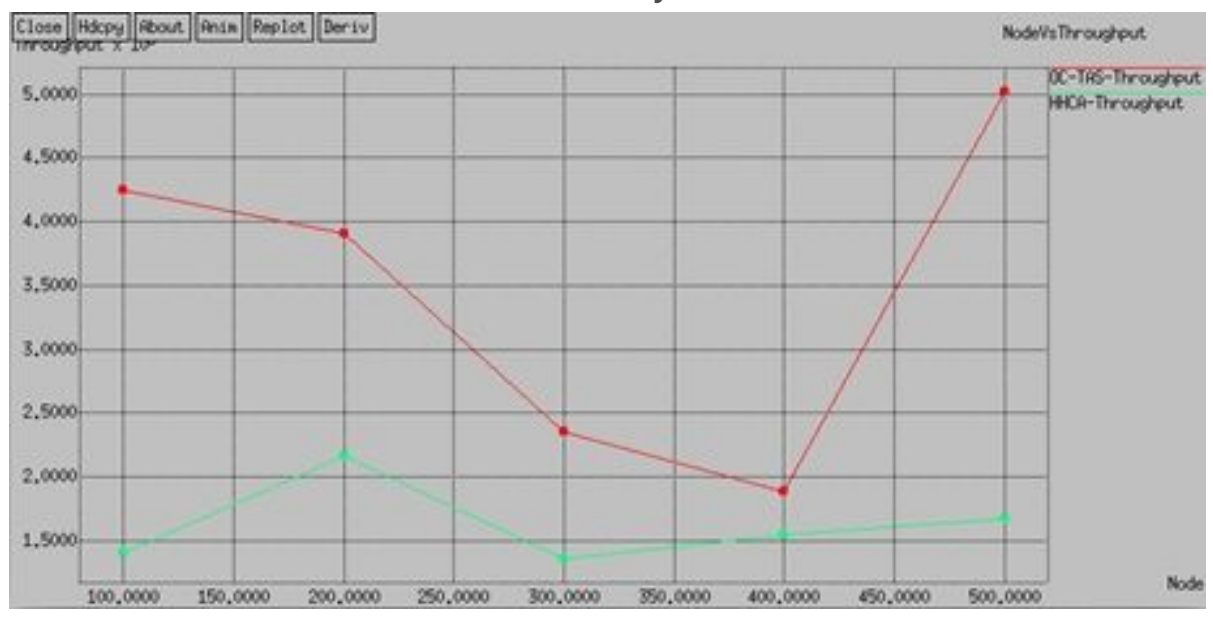

\section{Figure 8}

Network throughput with node density

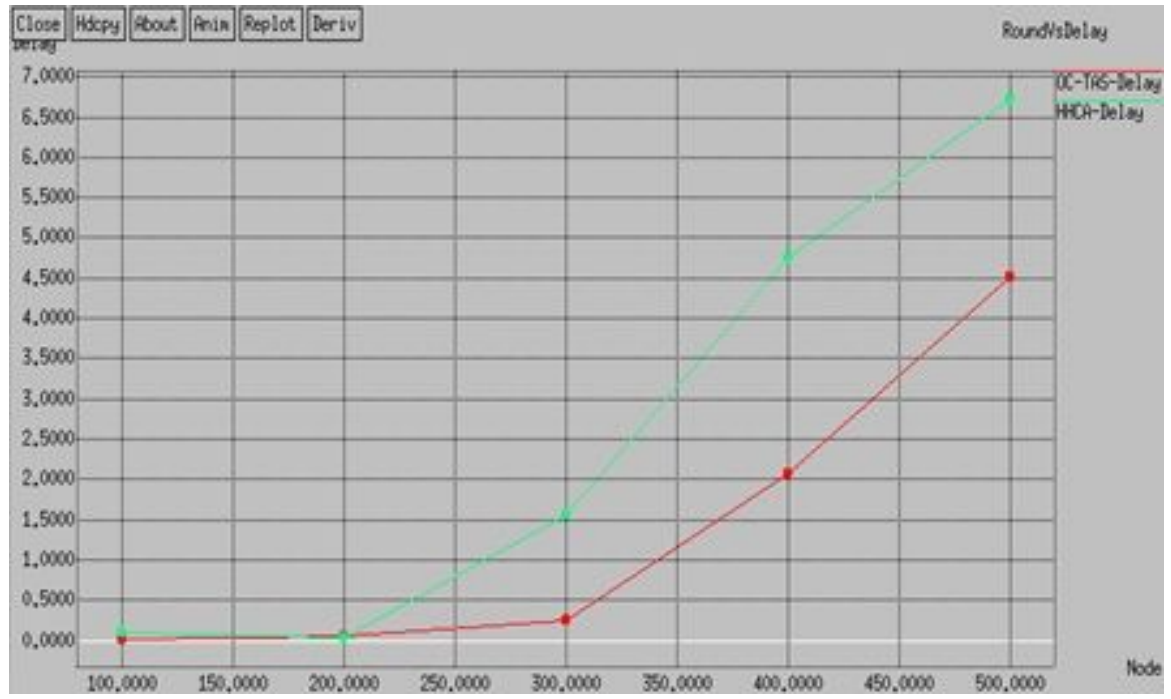

\section{Figure 9}

Delay comparison with number of rounds 


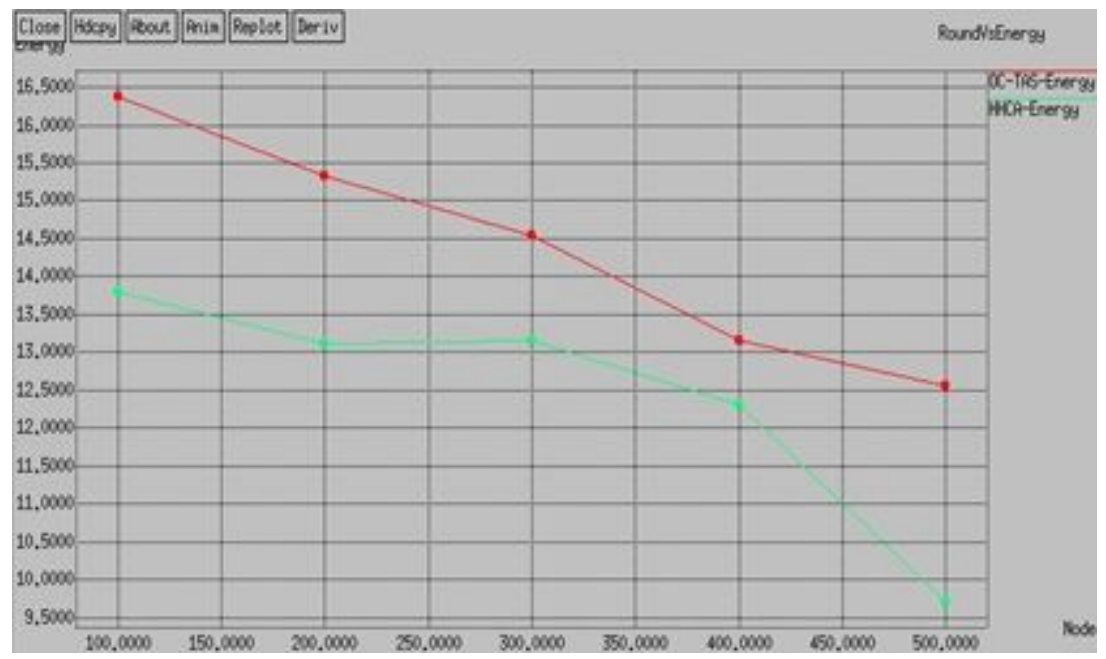

\section{Figure 10}

Energy consumption comparison with number of rounds

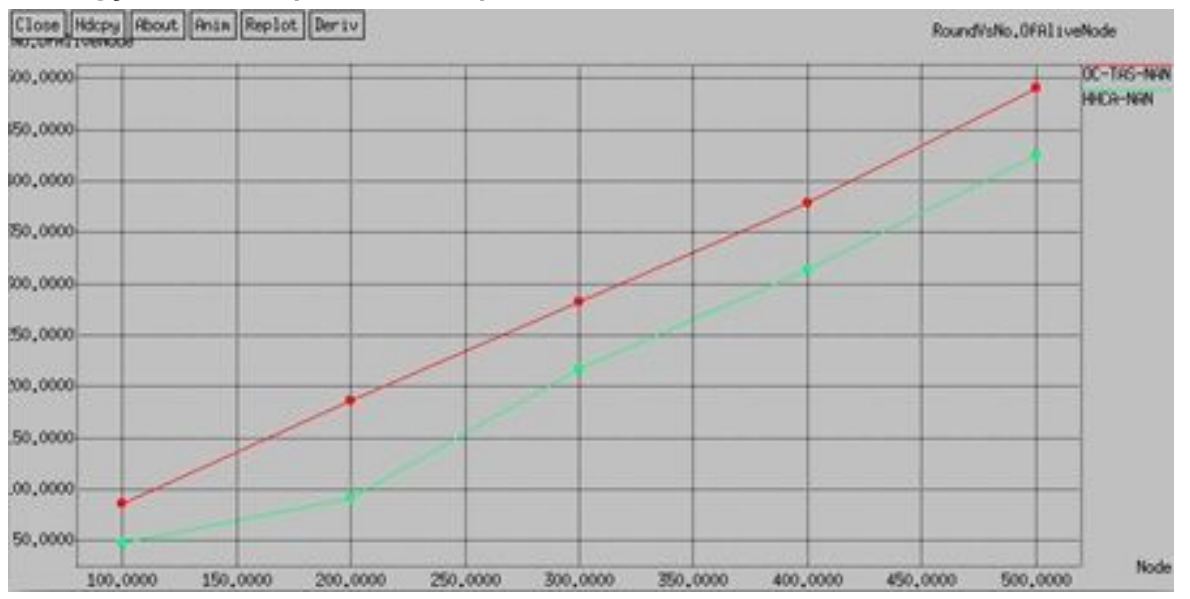

\section{Figure 11}

Number of alive nodes comparison with number of rounds

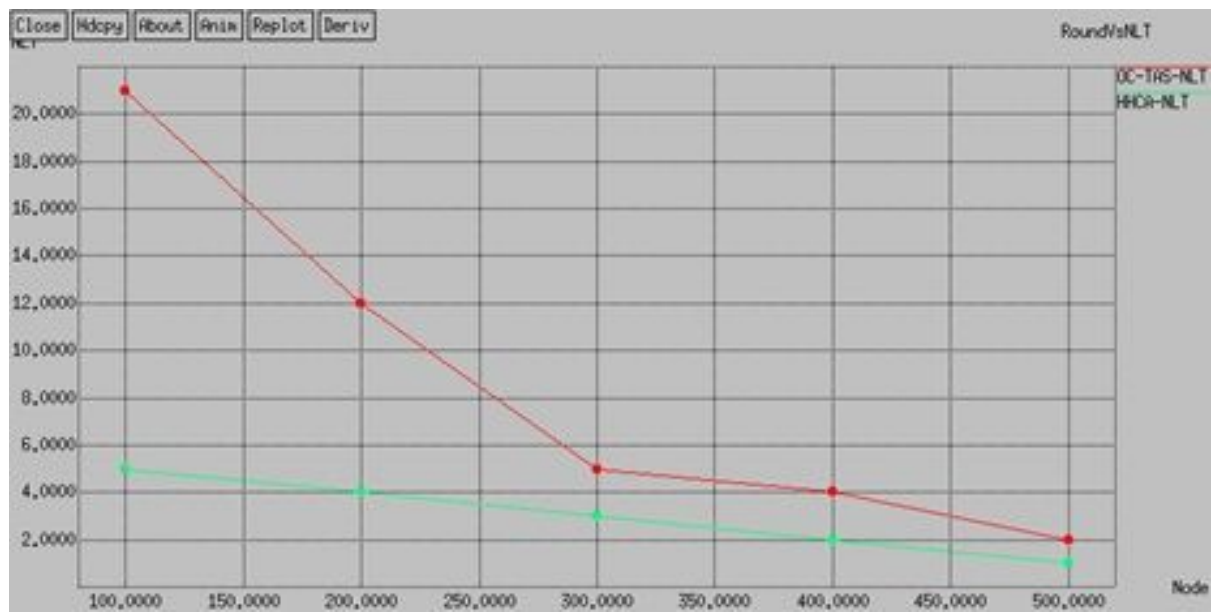

Figure 12 
Network lifetime comparison with number of rounds

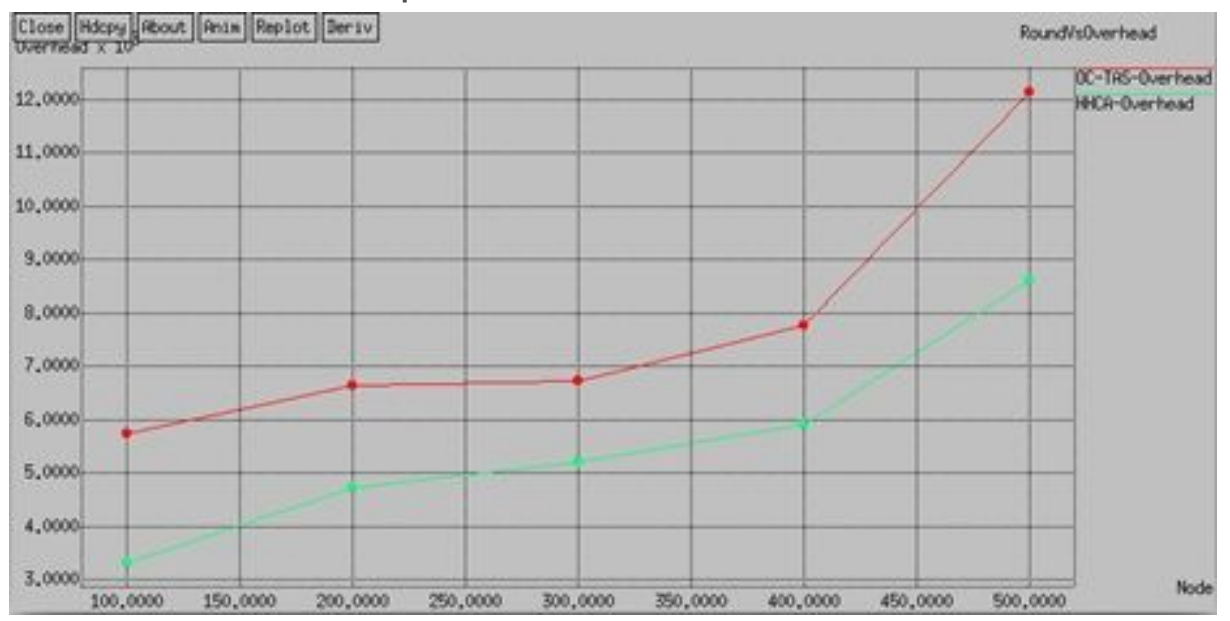

Figure 13

Network overhead comparison with number of rounds

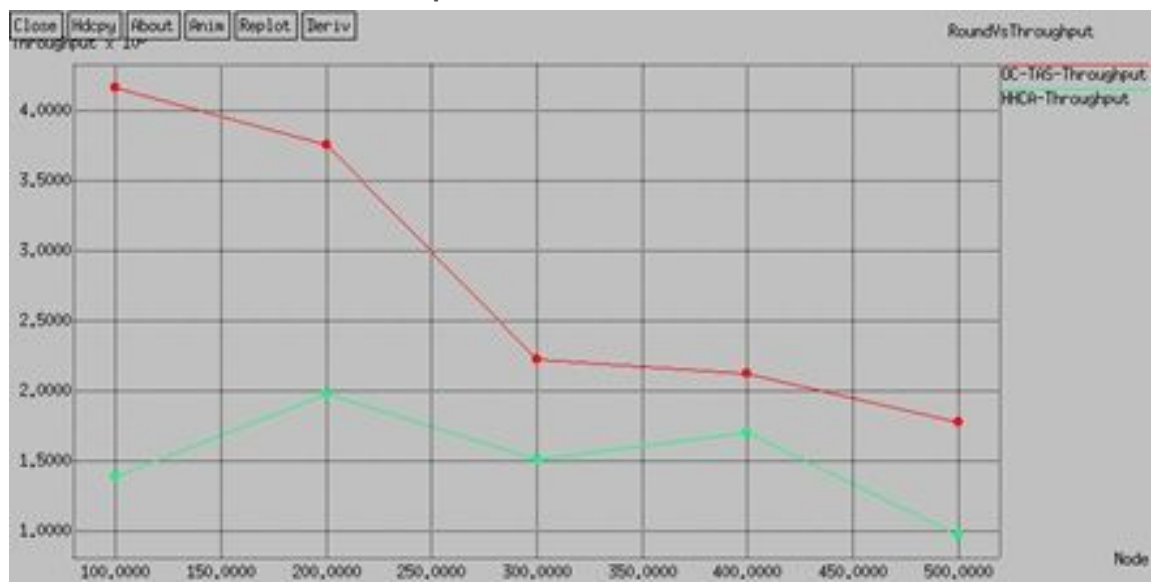

Figure 14

Network throughput comparison with number of rounds 